\title{
On convergence to equilibrium for one-dimensional chain of harmonic oscillators on the half-line
}

\author{
T.V. Dudnikova \\ Keldysh Institute of Applied Mathematics, \\ Miuskaya sq. 4, Moscow 125047, Russia \\ E-mail: tdudnikov@mail.ru
}

\begin{abstract}
The initial-boundary value problem for an infinite one-dimensional chain of harmonic oscillators on the half-line is considered. The large time asymptotic behavior of solutions is studied. The initial data of the system are supposed to be a random function which has some mixing properties. We study the distribution $\mu_{t}$ of the random solution at time moments $t \in \mathbb{R}$. The main result is the convergence of $\mu_{t}$ to a Gaussian probability measure as $t \rightarrow \infty$. We find stationary states in which there is a non-zero energy current at origin.
\end{abstract}

Key words and phrases: one-dimensional system of harmonic oscillators on the halfline; random initial data; mixing condition; Volterra integro-differential equation; compactness of measures; convergence to statistical equilibrium; energy current 


\section{Introduction}

We consider the infinite system of harmonic oscillators on the half-line:

$$
\ddot{u}(x, t)=\left(\Delta_{L}-m^{2}\right) u(x, t), \quad x \in \mathbb{N}, \quad t>0,
$$

with the boundary condition (as $x=0$ )

$$
\ddot{u}(0, t)=F(u(0, t))-m^{2} u(0, t)-\gamma \dot{u}(0, t)+u(1, t)-u(0, t), \quad t>0,
$$

and with the initial condition (as $t=0$ )

$$
u(x, 0)=u_{0}(x), \quad \dot{u}(x, 0)=v_{0}(x), \quad x \in \mathbb{Z}_{+} .
$$

Here $u(x, t) \in \mathbb{R}, m \geq 0, \gamma \geq 0, \mathbb{Z}_{+}=\{0,1,2,3, \ldots\}=0 \cup \mathbb{N}, \Delta_{L}$ denotes the second derivative on $\mathbb{Z}$ :

$$
\Delta_{L} u(x)=u(x+1)-2 u(x)+u(x-1), \quad x \in \mathbb{Z} .
$$

If $\gamma=0$, then formally system (1.1), (1.2) is Hamiltonian with the Hamiltonian functional

$$
\mathrm{H}(u, \dot{u}):=\frac{1}{2} \sum_{x \in \mathbb{Z}_{+}}\left(|\dot{u}(x, t)|^{2}+|u(x+1, t)-u(x, t)|^{2}+m^{2}|u(x, t)|^{2}\right)+P(u(0, t)),
$$

where, by definition, $P(q):=-\int F(q) d q, q \in \mathbb{R}$. To prove the existence of solutions to the problem (1.1) $-(1.3)$, we assume that $P \equiv 0$ or

$$
P \in C^{2}(\mathbb{R}), \quad P(q) \rightarrow+\infty \quad \text { as }|q| \rightarrow \infty,
$$

so $P(q) \geq P_{0}$ for all $q$ with some $P_{0} \in \mathbb{R}$.

Write $Y(t)=\left(Y^{0}(t), Y^{1}(t)\right) \equiv(u(\cdot, t), \dot{u}(\cdot, t)), Y_{0}=\left(Y_{0}^{0}, Y_{0}^{1}\right) \equiv\left(u_{0}(\cdot), v_{0}(\cdot)\right)$. We assume that the initial state $Y_{0}(x)$ belongs to the Hilbert space $\mathcal{H}_{\alpha,+}, \alpha \in \mathbb{R}$, consisting of real sequences, see Definition 2.1 below. The existence and uniqueness of the solutions $Y(t)$ is proved in Appendix A.

To prove the main result we assume that $F(q)=-\kappa q$ with $\kappa \geq 0$. Moreover, we impose some restrictions on the coefficients $m, \kappa, \gamma$ of the system, see condition $\mathbf{C}$ below. In particular, if $\gamma \neq 0$, then at least one of the numbers $m$ and $\kappa$ is not zero. If $\gamma=0$, then $\kappa \in(0,2)$. The initial state $Y_{0}(x)$ is supposed to be a random element of the space $\mathcal{H}_{\alpha,+}, \alpha<-3 / 2$, with the distribution $\mu_{0}$. We assume that $\mu_{0}$ is a probability measure of mean zero satisfying conditions S2-S4. In particular, the initial measure $\mu_{0}$ satisfies a mixing condition. Roughly speaking, it means that $Y_{0}(x)$ and $Y_{0}(y)$ are asymptotically independent as $|x-y| \rightarrow \infty$.

For a given $t \in \mathbb{R}$, denote by $\mu_{t}$ the probability measure on $\mathcal{H}_{\alpha,+}$ giving the distribution of the random solution $Y(t)=(u(\cdot, t), \dot{u}(\cdot, t))$ to the problem (1.1)-(1.3). Our main objective is to prove the weak convergence of the measures $\mu_{t}$ on the space $\mathcal{H}_{\alpha,+}$ with $\alpha<-3 / 2$ to a limit measure $\mu_{\infty}$, which is an equilibrium Gaussian measure on $\mathcal{H}_{\alpha,+}$,

$$
\mu_{t} \rightarrow \mu_{\infty} \quad \text { as } t \rightarrow \infty
$$

This means the convergence of the integrals $\int f(Y) \mu_{t}(d Y) \rightarrow \int f(Y) \mu_{\infty}(d Y)$ as $t \rightarrow \infty$ for any bounded continuous functional $f$ on $\mathcal{H}_{\alpha,+}$. Furthermore, we find stationary states $\mu_{\infty}$ in which there is a non-zero energy current at the origin, see Remark 2.11 below. 
For one-dimensional chains of harmonic oscillators in the whole line, the convergence to equilibrium distribution has been established by Boldrighini et al. [1] and by Spohn and Lebowitz [20]. Ergodic properties of one-dimensional chains of anharmonic oscillators coupled to heat baths were studied by Jakšić, Pillet and others (see, e.g., [11, 12, 7]). The convergence (1.6) was proved also for harmonic crystals in $\mathbb{Z}^{d}$ with $d \geq 1$ [2, 3] and for a scalar KleinGordon field coupled to a harmonic crystal [4]. Similar results were obtained in [5] for the harmonic crystals in the half-space $\mathbb{Z}_{+}^{d}:=\left\{x=\left(x_{1}, \ldots, x_{d}\right) \in \mathbb{Z}^{d}: x_{1} \geq 0\right\}$ with zero boundary condition. In the present paper, we treat the one-dimensional model but with the boundary condition of the form (1.2).

We outline the strategy of the proof. Using the technique of [2, 5], we derive the convergence (1.6) from the assertions I and II:

I. The family of measures $\mu_{t}, t \geq 0$, is weakly compact in $\mathcal{H}_{\alpha,+}$ with $\alpha<-3 / 2$.

II. The characteristic functionals of $\mu_{t}$ converge to a Gaussian functional,

$$
\hat{\mu}_{t}(\Psi):=\int \exp \left(i\langle Y, \Psi\rangle_{+}\right) \mu_{t}(d Y) \rightarrow \exp \left\{-\frac{1}{2} \mathcal{Q}_{\infty}(\Psi, \Psi)\right\}, \quad t \rightarrow \infty .
$$

Here $\Psi=\left(\Psi^{0}, \Psi^{1}\right) \in \mathcal{S}:=S \oplus S$, where $S$ denotes a space of real rapidly decreasing sequences,

$$
\langle Y, \Psi\rangle_{+}=\sum_{i=0,1} \sum_{x \in \mathbb{Z}_{+}} Y^{i}(x) \Psi^{i}(x) \quad \text { for } Y=\left(Y^{0}, Y^{1}\right) \in \mathcal{H}_{\alpha,+} \quad \text { and } \Psi=\left(\Psi^{0}, \Psi^{1}\right) \in \mathcal{S},
$$

$\mathcal{Q}_{\infty}(\Psi, \Psi)$ is a quadratic form.

To prove I we derive the uniform bound (5.1) for the mean local energy with respect to the measure $\mu_{t}, t \geq 0$, and apply the Prokhorov compactness theorem. To check II we study the asymptotic behavior of the solution $Y(t)$ and obtain (see Lemma 4.7) that

$$
\langle Y(t), \Psi\rangle_{+} \sim\left\langle U_{0}(t) Y_{0}, \Pi_{\Psi}\right\rangle, \quad t \rightarrow \infty \quad \text { (in mean) }
$$

where $U_{0}(t)$ is a solving operator to the problem (1.1) and (1.3) with zero boundary condition (i.e., $u(0, t) \equiv 0)$, the functions $\Pi_{\Psi}$ are expressed using $\Psi \in \mathcal{S}$ (see formula (4.22)).

To prove (1.8) we decompose the solution $u(x, t)$ into two terms: $u(x, t)=z(x, t)+q(x, t)$. Here $z(x, t)$ is a solution of (1.1) satisfying zero boundary condition and the initial condition (1.3) , i.e., $(z(\cdot, t), \dot{z}(\cdot, t))=U_{0}(t) Y_{0} . q(x, t)$ is a solution of (1.1) with zero initial condition for $x \neq 0$ and with the boundary condition (2.7). The existence and behavior of the solution $z(x, t)$ were studied in [5]. We state the basic results on $z(x, t)$ in Section 2.3. For $q(x, t)$ with $x \neq 0$, the following representation holds,

$$
q(x, t)=\int_{0}^{t} K(x, t-s) q(0, s) d s, \quad x \in \mathbb{N},
$$

where the kernel $K(x, t)$ is defined in (3.5). The properties of this kernel are studied in Appendix A. In particular, $K(x, t)$ satisfies the following bound:

$$
\sum_{x \in \mathbb{N}}\left(1+x^{2}\right)^{\alpha}|K(x, t)|^{2} \sim C(1+|t|)^{-3} \quad \text { as } t \rightarrow \infty, \quad \text { for any } \alpha<-3 / 2 .
$$

Therefore, to prove (1.8) it remains to study the long time behavior of $q(x, t)$ with $x=0$. By (1.2) and (1.9),$q(0, t)$ evolves according to a Volterra integro-differential equation of the form

$$
\ddot{q}(0, t)=F(q(0, t))-\left(1+m^{2}\right) q(0, t)-\gamma \dot{q}(0, t)+\int_{0}^{t} K(1, t-s) q(0, s) d s+h(t), \quad t>0,
$$


with $h(t)=z(1, t)$. To investigate the solutions of (1.11), we assume that $F(q)=-\kappa q$ with $\kappa \geq 0$. This allows us to apply the Fourier-Laplace transform $t \rightarrow \omega$ to the solutions $q(0, t)$ and study the analytic properties of $\tilde{q}(0, \omega)$ for complex values $\omega \in \mathbb{C}$ (see Appendix B). These properties give the bound for the solutions $q(0, t)$ of (1.11) with $h(t) \equiv 0$ :

$$
|q(0, t)| \leq C(1+t)^{-3 / 2}, \quad t \geq 0 .
$$

Applying this estimate together with (1.9) and (1.10), we obtain the asymptotics for $q(x, t)$ in mean (see formula (4.19) below)

$$
\langle q(\cdot, t), \psi\rangle_{+} \sim\left\langle U_{0}(t) Y_{0}, \mathbf{K}_{\psi}^{0}\right\rangle_{+}, \quad t \rightarrow \infty
$$

where $\psi \in S$, the vector valued function $\mathbf{K}_{\psi}^{0}$ is defined in (4.18). This implies the asymptotics (1.8) which plays the crucial role in our convergence analysis for the statistical solutions of the problem (1.1)-(1.3)

The dynamics of the equations with delay has been extensively investigated by many authors under some restrictions on the kernel $K(1, t)$. For details, we refer to the monograph by Gripenberg, Londen and Staffans [8]. The stability properties of linear Volterra integrodifferential equations can be found in the papers by Murakami [16], Nino and Murakami [17], Kordonis and Philos [15]. Note that in the literature frequently the long time asymptotical behavior of solutions is studied assuming that the kernel has the exponential decay or is of one sign. However, in our case, by (1.10), the decay of $K(1, t)$ is like $(1+t)^{-3 / 2}$.

The nonlinear equations of the form (1.11) with a stationary Gaussian process $h(t)$ and with a smooth (confining or periodic) potential $P(q)=-\int F(q) d q$ have been investigated extensively, for example, the ergodic properties of such equations were studied by Jakšić and Pillet in [11, and the qualitative properties of solutions were established by Ottobre and Pavliotis in [18]. In the present paper, we prove the convergence to equilibrium for a linear model. However, we do not assume that the initial distribution of the system is a Gibbs measure or absolutely continuous with respect to a Gibbs measure. Therefore, the force $h(t)=z(1, t)$ in (1.11) is non-Gaussian, in general.

The paper is organized as follows. In Section 2, we impose the conditions on the model and on the initial measures $\mu_{0}$ and state the main results. The bounds for solutions of (1.11) are obtained in Section 3. The asymptotics (1.8) is proved in Section 4. In Section 5, we prove the convergence (1.6). In Appendix A, we study the properties of the kernel $K(x, t)$ and prove the existence of the solutions. The properties of $\tilde{q}(0, \omega), \omega \in \mathbb{C}$, are studied in Appendix B. Appendix $\mathrm{C}$ contains the results on the solutions $z(x, t)$.

\section{Main Results}

\subsection{Phase space}

We assume that the initial date $Y_{0}$ belongs to the phase space $\mathcal{H}_{\alpha,+}, \alpha \in \mathbb{R}$, defined below.

Definition 2.1 (i) $\ell_{\alpha,+}^{2} \equiv \ell_{\alpha,+}^{2}\left(\mathbb{Z}_{+}\right), \alpha \in \mathbb{R}$, is the Hilbert space of sequences $u(x), x \in \mathbb{Z}_{+}$, with the norm

$$
\|u\|_{\alpha,+}^{2}=\sum_{x \in \mathbb{Z}_{+}}|u(x)|^{2}\langle x\rangle^{2 \alpha}<\infty, \quad\langle x\rangle:=\left(1+x^{2}\right)^{1 / 2} .
$$


(ii) $\mathcal{H}_{\alpha,+}=\ell_{\alpha,+}^{2} \otimes \ell_{\alpha,+}^{2}$ is the Hilbert space of pairs $Y(x)=(u(x), v(x))$ of sequences equipped with the norm $\|Y\|_{\alpha,+}^{2}=\|u\|_{\alpha,+}^{2}+\|v\|_{\alpha,+}^{2}<\infty$.

(iii) $\ell_{\alpha}^{2} \equiv \ell_{\alpha}^{2}(\mathbb{Z})$ is the Hilbert space of sequences with the norm $\|u\|_{\alpha}^{2}=\sum_{x \in \mathbb{Z}}|u(x)|^{2}\langle x\rangle^{2 \alpha}<\infty$. In particular, $\ell_{0}^{2} \equiv \ell^{2}$. Write $\mathcal{H}_{\alpha}:=\ell_{\alpha}^{2} \otimes \ell_{\alpha}^{2}, \alpha \in \mathbb{R}$.

Theorem 2.2 Let $\gamma, m \geq 0$ and condition (1.5) hold, and let $Y_{0} \in \mathcal{H}_{\alpha,+}, \alpha \in \mathbb{R}$. Then the problem (1.1)-(1.3) has a unique solution $Y(t) \in C\left(\mathbb{R}, \mathcal{H}_{\alpha,+}\right)$. The operator $U(t): Y_{0} \rightarrow Y(t)$ is continuous on $\mathcal{H}_{\alpha,+}$. Moreover, there exist constants $C, B<\infty$ such that $\left\|U(t) Y_{0}\right\|_{\alpha,+} \leq$ $C e^{B|t|}$, where the constant $C$ depends on $\left\|Y_{0}\right\|_{\alpha,+}$.

Theorem 2.2 is proved in Appendix A. The proof is based on the following representation for the solution $u(x, t)$ of the problem (1.1)-(1.3):

$$
u(x, t)=z(x, t)+q(x, t), \quad x \in \mathbb{Z}_{+}, \quad t>0,
$$

where $z(x, t)$ is a solution of the initial-boundary value problem with zero boundary condition,

$$
\begin{aligned}
& \ddot{z}(x, t)=\left(\Delta_{L}-m^{2}\right) z(x, t), \quad x \in \mathbb{N}, \quad t>0, \\
& z(0, t)=0, \quad t \geq 0, \\
& z(x, 0)=u_{0}(x), \quad \dot{z}(x, 0)=v_{0}(x), \quad x \in \mathbb{N} .
\end{aligned}
$$

Therefore, $q(x, t)$ is a solution of the following initial-boundary value problem

$$
\begin{aligned}
& \ddot{q}(x, t)=\left(\Delta_{L}-m^{2}\right) q(x, t), \quad x \in \mathbb{N}, \quad t>0, \\
& \ddot{q}(0, t)=F(q(0, t))-m^{2} q(0, t)-\gamma \dot{q}(0, t)+q(1, t)-q(0, t)+z(1, t), t>0, \\
& q(x, 0)=0, \quad \dot{q}(x, 0)=0, \quad x \in \mathbb{N}, \\
& q(0,0)=u_{0}(0), \quad \dot{q}(0,0)=v_{0}(0) .
\end{aligned}
$$

In Section 2.3, we state the properties of the solutions $z(x, t)$. In Section 3 , we study the behavior of $q(x, t)$.

To prove the main result we assume that $F(q)=-\kappa q$, with $\kappa \geq 0$. Moreover, on the nonnegative constants $m, \gamma, \kappa$ of the system we impose the following condition $\mathbf{C}$.

C If $\gamma \neq 0$, then either $m \neq 0$ or $\kappa \neq 0$. In addition, if $\gamma \in(0,1)$ and $m=0$, then $\kappa \neq 2\left(1-\gamma^{2}\right)$; if $\gamma \in\left(0,\left(\sqrt{m^{2}+4}-m\right) / 2\right]$ and $m \neq 0$, then $\kappa \neq 1-\gamma^{2} \pm$ $\sqrt{\left(1-\gamma^{2}\right)^{2}-m^{2} \gamma^{2}}$. If $\gamma=0$, then $\kappa \in(0,2)$.

\subsection{Random initial data}

We assume that the initial date $Y_{0}(x)=\left(Y_{0}^{0}(x), Y_{0}^{1}(x)\right)$ is a measurable random function with values in $\left(\mathcal{H}_{\alpha,+}, \mathcal{B}\left(\mathcal{H}_{\alpha,+}\right)\right)$, where $\mathcal{B}\left(\mathcal{H}_{\alpha,+}\right)$ stands for the Borel $\sigma$-algebra in $\mathcal{H}_{\alpha,+}$. Denote by $\mu_{0}$ a Borel probability measure on $\mathcal{H}_{\alpha,+}$ giving the distribution of $Y_{0}$. The expectation with respect to $\mu_{0}$ is denoted by $\mathbb{E}$. We impose the following conditions $\mathbf{S} 1-\mathbf{S} 4$ on $\mu_{0}$.

S1 The measure $\mu_{0}$ has zero expectation value, $\mathbb{E}\left(Y_{0}(x)\right) \equiv \int Y_{0}(x) \mu_{0}\left(d Y_{0}\right)=0, x \in \mathbb{Z}_{+}$.

S2 The measure $\mu_{0}$ has finite variance,

$$
\sup _{x \in \mathbb{Z}_{+}} \mathbb{E}\left(\left|Y_{0}(x)\right|^{2}\right) \leq e_{0}<\infty
$$


Write $\nu_{0}=\mu_{0}\left\{Y_{0} \in \mathcal{H}_{\alpha,+}: Y_{0}(0)=0\right\}$. The expectation with respect to $\nu_{0}$ is denoted by $\mathbb{E}_{0}$. The correlation functions of the measure $\nu_{0}$ are denoted by

$$
Q_{0}^{i j}\left(x, x^{\prime}\right)=\mathbb{E}_{0}\left(Y_{0}^{i}(x) Y_{0}^{j}\left(x^{\prime}\right)\right), \quad x, x^{\prime} \in \mathbb{Z}_{+}, \quad i, j=0,1
$$

In particular, the matrix $Q_{0}\left(x, x^{\prime}\right)=\left(Q_{0}^{i j}\left(x, x^{\prime}\right)\right)_{i, j=0,1}$ vanishes if $x=0$ or $x^{\prime}=0$. On the measure $\nu_{0}$ we impose conditions $\mathbf{S 3}$ and $\mathbf{S 4}$.

S3 For every $x \in \mathbb{Z}, \lim _{y \rightarrow+\infty} Q_{0}^{i j}(x+y, y)=q_{0}^{i j}(x), i, j=0,1$. Here $q_{0}^{i j}(x)$ are correlation functions of some translation invariant measure $\nu$ with zero mean value on the space $\mathcal{H}_{\alpha}$.

By definition, a measure $\nu$ is said to be translation invariant if $\nu\left(T_{h} B\right)=\nu(B)$ for any $B \in \mathcal{B}\left(\mathcal{H}_{\alpha}\right)$ and $h \in \mathbb{Z}$, where $T_{h} Y_{0}(x)=Y_{0}(x-h)$ for $x \in \mathbb{Z}$.

To formulate the last condition on $\nu_{0}$, denote by $\mathcal{A}$ an open interval of $\mathbb{Z}_{+}$and by $\sigma(\mathcal{A})$ the $\sigma$-algebra on $\mathcal{H}_{\alpha,+}$ generated by $Y_{0}(x)$ with $x \in \mathcal{A}$. Define the Ibragimov mixing coefficient of a probability measure $\nu$ on $\mathcal{H}_{\alpha,+}$ by the rule (cf [9, Definition 17.2.2])

$$
\varphi(r) \equiv \sup _{\substack{\mathcal{A}, \mathcal{B} \subset \mathbb{Z}_{+} \\ \operatorname{dist}(\mathcal{A}, \mathcal{B}) \geq r}} \sup _{\substack{A \in(\mathcal{A}), B \in \sigma(\mathcal{B}) \\ \nu(B)>0}}
$$

A measure $\nu$ is said to satisfy the strong uniform Ibragimov mixing condition if $\varphi(r) \rightarrow 0$ as $r \rightarrow \infty$.

S4 The measure $\nu_{0}$ satisfies the strong uniform Ibragimov mixing condition with mixing coefficient $\varphi$, and $\int_{0}^{+\infty} \varphi^{1 / 2}(r) d r<\infty$.

The examples of the initial measures $\nu_{0}$ satisfying conditions $\mathbf{S 3}$ and $\mathbf{S} 4$ are given in [5].

Lemma 2.3 (see [5, Proposition 5.2]) Let conditions $\mathbf{S 1 - S 4 ~ h o l d . ~ T h e n ~} q_{0}^{i j} \in \ell^{1}$. Moreover, for any $\Phi, \Psi \in \mathcal{H}_{0,+}$, the following bound holds

$$
\left|\left\langle Q_{0}\left(z, z^{\prime}\right), \Phi(z) \otimes \Psi\left(z^{\prime}\right)\right\rangle_{+}\right| \leq C\|\Phi\|_{0,+}\|\Psi\|_{0,+} .
$$

This implies, in particular, that $\hat{q}_{0}^{i j} \in C(\mathbb{T})$. Here and below $\hat{q}(\theta) \equiv F_{x \rightarrow \theta}[q(x)]$ denotes the discrete Fourier transform w.r.t. $x \in \mathbb{Z}$,

$$
\hat{q}(\theta)=\sum_{x \in \mathbb{Z}} e^{i x \theta} q(x), \quad \theta \in \mathbb{T} \equiv \mathbb{R} / 2 \pi \mathbb{Z}
$$

For a probability measure $\nu$ on $\mathcal{H}_{\alpha,+}$ denote by $\hat{\nu}$ the characteristic functional (Fourier transform)

$$
\hat{\nu}(\Psi) \equiv \int \exp \left(i\langle Y, \Psi\rangle_{+}\right) \nu(d Y), \quad \Psi \in \mathcal{S} .
$$

A measure $\nu$ is called Gaussian (with zero expectation) if its characteristic functional has the form $\hat{\nu}(\Psi)=\exp \{-\mathcal{Q}(\Psi, \Psi) / 2\}, \Psi \in \mathcal{S}$, where $\mathcal{Q}$ is a real nonnegative quadratic form in $\mathcal{S}$. 


\subsection{Convergence to equilibrium for the problem $(\underline{2.2})-(\underline{2.4})$}

In this section we state the results concerning the solutions of the problem (2.2)-(2.4) (see [5]). Write $Z(t) \equiv Z(x, t)=(z(x, t), \dot{z}(x, t))$.

Lemma 2.4 (see Lemma 2.7 in [5]) Assume that $\alpha \in \mathbb{R}$. Then (i) for any $Y_{0} \in \mathcal{H}_{\alpha,+}$, there exists a unique solution $Z(t) \in C\left(\mathbb{R}, \mathcal{H}_{\alpha,+}\right)$ to the problem (2.2)-2.4); (ii) the operator $U_{0}(t): Y_{0} \mapsto Z(t)$ is continuous on $\mathcal{H}_{\alpha,+}$.

The proof is based on the following formula for the solution $Z(x, t)$ of the problem (2.2)(2.4):

$$
Z^{i}(x, t)=\sum_{j=0,1} \sum_{x^{\prime} \in \mathbb{N}} \mathcal{G}_{t,+}^{i j}\left(x, x^{\prime}\right) Y_{0}^{j}\left(x^{\prime}\right), \quad x \in \mathbb{Z}_{+},
$$

where $Z(x, t)=\left(Z^{0}(x, t), Z^{1}(x, t)\right) \equiv(z(x, t), \dot{z}(x, t))$, the Green function $\mathcal{G}_{t,+}\left(x, x^{\prime}\right)$ is

$$
\mathcal{G}_{t,+}\left(x, x^{\prime}\right):=\mathcal{G}_{t}\left(x-x^{\prime}\right)-\mathcal{G}_{t}\left(x+x^{\prime}\right), \quad \mathcal{G}_{t}(x) \equiv \frac{1}{2 \pi} \int_{\mathbb{T}} e^{-i x \theta} \hat{\mathcal{G}}_{t}(\theta) d \theta, \quad x \in \mathbb{Z},
$$

with

$$
\hat{\mathcal{G}}_{t}(\theta)=\left(\hat{\mathcal{G}}_{t}^{i j}(\theta)\right)_{i, j=0}^{1}=\left(\begin{array}{ll}
\cos \phi(\theta) t & \frac{\sin \phi(\theta) t}{\phi(\theta)} \\
-\phi(\theta) \sin \phi(\theta) t & \cos \phi(\theta) t
\end{array}\right), \quad \phi(\theta)=\sqrt{2-2 \cos \theta+m^{2}} .
$$

In particular, $\phi(\theta)=2|\sin (\theta / 2)|$ if $m=0$. We see that $Z(0, t) \equiv 0 \forall t$, since $\mathcal{G}_{t}(-x)=\mathcal{G}_{t}(x)$.

Definition 2.5 We define $\nu_{t}$ as the Borel probability measure on $\mathcal{H}_{\alpha,+}$, which gives the distribution of the solution $Z(t)$ to the problem (2.2)-(2.4), i.e., $\nu_{t}(B)=\nu_{0}\left(U_{0}(-t) B\right)$ for any $B \in \mathcal{B}\left(\mathcal{H}_{\alpha,+}\right), t \in \mathbb{R}$.

Lemma 2.6 (see [5, Theorem A]) The correlation functions of $\nu_{t}$ converge to a limit,

$$
Q_{t}^{i j}\left(x, x^{\prime}\right)=\int Z^{i}(x) Z^{j}\left(x^{\prime}\right) \nu_{t}(d Z) \rightarrow Q_{\infty}^{i j}\left(x, x^{\prime}\right), \quad t \rightarrow \infty, \quad x, x^{\prime} \in \mathbb{Z}_{+}
$$

The correlation matrix $Q_{\infty}\left(x, x^{\prime}\right)=\left(Q_{\infty}^{i j}\left(x, x^{\prime}\right)\right)_{i, j=0}^{1}$ has the form

$$
Q_{\infty}\left(x, x^{\prime}\right)=q_{\infty}\left(x-x^{\prime}\right)-q_{\infty}\left(x+x^{\prime}\right)-q_{\infty}\left(-x-x^{\prime}\right)+q_{\infty}\left(-x+x^{\prime}\right), \quad x, x^{\prime} \in \mathbb{Z}_{+} .
$$

Here $q_{\infty}(x)=q_{\infty}^{+}(x)+q_{\infty}^{-}(x), x \in \mathbb{Z}$, where in the Fourier transform we have

$$
\begin{aligned}
& \hat{q}_{\infty}^{+}(\theta)=\frac{1}{2}\left(\begin{array}{cc}
\hat{q}_{0}^{00}(\theta)+\hat{q}_{0}^{11}(\theta) \phi^{-2}(\theta) & \hat{q}_{0}^{01}(\theta)-\hat{q}_{0}^{10}(\theta) \\
-\hat{q}_{0}^{01}(\theta)+\hat{q}_{0}^{10}(\theta) & \phi^{2}(\theta) \hat{q}_{0}^{00}(\theta)+\hat{q}_{0}^{11}(\theta)
\end{array}\right), \\
& \hat{q}_{\infty}^{-}(\theta)=\frac{1}{2} \operatorname{sign}(\theta)\left(\begin{array}{cc}
\left(\hat{q}_{0}^{10}(\theta)-\hat{q}_{0}^{01}(\theta)\right) \phi^{-1}(\theta) & \hat{q}_{0}^{00}(\theta) \phi(\theta)+\hat{q}_{0}^{11}(\theta) \phi^{-1}(\theta) \\
-\hat{q}_{0}^{00}(\theta) \phi(\theta)-\hat{q}_{0}^{11}(\theta) \phi^{-1}(\theta) & \left(\hat{q}_{0}^{10}(\theta)-\hat{q}_{0}^{01}(\theta)\right) \phi(\theta)
\end{array}\right) .
\end{aligned}
$$

Here $\theta \in \mathbb{T}$ if $m \neq 0$ and $\theta \in \mathbb{T} \backslash\{0\}$ if $m=0$, the functions $q_{0}^{i j}, i, j=0,1$, are introduced in condition $\mathbf{S 3}, \phi(\theta)$ is defined in (2.12). 
Denote by $\mathcal{Q}_{\infty}^{\nu}(\Psi, \Psi)$ a real quadratic form on $\mathcal{S}$ defined by

$$
\mathcal{Q}_{\infty}^{\nu}(\Psi, \Psi)=\left\langle Q_{\infty}\left(x, x^{\prime}\right), \Psi(x) \otimes \Psi\left(x^{\prime}\right)\right\rangle_{+} \equiv \sum_{i, j=0,1} \sum_{x, x^{\prime} \in \mathbb{Z}_{+}} Q_{\infty}^{i j}\left(x, x^{\prime}\right) \Psi^{i}(x) \Psi^{j}\left(x^{\prime}\right)
$$

Remark 2.7 Given $\Psi(x), x \in \mathbb{Z}_{+}$, introduce an odd sequence $\Psi_{o}(x), x \in \mathbb{Z}$, such that $\Psi_{o}(x)=\Psi(x)$ for $x>0, \Psi_{o}(0)=0$ and $\Psi_{o}(x)=-\Psi(-x)$ for $x<0$. Note that $Q_{\infty}\left(x, x^{\prime}\right)$ is odd w.r.t. $x$ and $x^{\prime}$. Moreover,

$$
\mathcal{Q}_{\infty}^{\nu}(\Psi, \Psi)=\left\langle q_{\infty}\left(x-x^{\prime}\right), \Psi_{o}(x) \otimes \Psi_{o}\left(x^{\prime}\right)\right\rangle \equiv \sum_{i, j=0}^{1} \sum_{x, x^{\prime} \in \mathbb{Z}} q_{\infty}^{i j}\left(x-x^{\prime}\right) \Psi_{o}^{i}(x) \Psi_{o}^{j}\left(x^{\prime}\right)
$$

In the case $m \neq 0, q_{\infty}^{i j} \in \ell^{1}$, by Lemma 2.3 and formulas (2.15). Then, Young's inequality yields

$$
\left|\mathcal{Q}_{\infty}^{\nu}(\Psi, \Psi)\right| \leq C\left\|q_{\infty}\right\|_{\ell^{1}}\left\|\Psi_{o}\right\|_{0}^{2} \leq C_{1}\left\|q_{\infty}\right\|_{\ell^{1}}\|\Psi\|_{0,+}^{2},
$$

i.e., $\mathcal{Q}_{\infty}^{\nu}(\Psi, \Psi)$ is continuous in $\mathcal{H}_{0,+} \equiv \ell_{0,+}^{2} \times \ell_{0,+}^{2}$. Here as before $\|\cdot\|_{0}\left(\|\cdot\|_{0,+}\right)$ stands for the norm in $\ell_{0}^{2} \equiv \ell^{2}$ and in $\mathcal{H}_{0}$ (in $\ell_{0,+}^{2}$ and in $\mathcal{H}_{0,+}$, respectively).

In the case $m=0, \phi(\theta)=2|\sin (\theta / 2)|$. Note that $\left|\hat{\Psi}_{o}(\theta)\right| \leq C(\Psi)|\sin \theta|$ for $\Psi \in S$. Denote $\ell_{1 / \phi,+}^{2}=\left\{\Psi \in \ell_{0,+}^{2}: \hat{\Psi}_{o} / \phi \in L^{2}(\mathbb{T})\right\}$ with norm $\|\Psi\|_{1 / \phi,+}:=\left\|(1+1 / \phi(\theta)) \hat{\Psi}_{o}(\theta)\right\|_{L^{2}(\mathbb{T})}$. Therefore, for any $\Psi=\left(\Psi^{0}, \Psi^{1}\right) \in \mathcal{H}_{1 / \phi,+}:=\ell_{1 / \phi,+}^{2} \times \ell_{0,+}^{2}$,

$$
\mathcal{Q}_{\infty}^{\nu}(\Psi, \Psi) \leq\left\|q_{0}\right\|_{\ell^{1}}\left(C_{1}\left\|F^{-1}(1 / \phi) * \Psi_{o}^{0}\right\|_{0}^{2}+C_{2}\left\|\Psi_{o}\right\|_{0}^{2}\right) \leq C_{3}\left\|\Psi^{0}\right\|_{1 / \phi,+}^{2}+C_{4}\left\|\Psi^{1}\right\|_{0,+}^{2},
$$

by (2.15) and (2.17). Here and below by $F^{-1}$ we denote the inverse Fourier transform.

Theorem 2.8 Let conditions $\mathbf{S 1 - S 4 ~ h o l d ~ a n d ~ l e t ~} \alpha<-1 / 2$ if $m \neq 0$ and $\alpha<-1$ if $m=0$. Then the following assertions are fulfilled.

(i) The measures $\nu_{t}$ weakly converge as $t \rightarrow \infty$ on the space $\mathcal{H}_{\alpha,+}$. The limit measure $\nu_{\infty}$ is Gaussian, with zero mean value and with correlation matrix $Q_{\infty}$ defined in (2.14).

(ii) The uniform bound holds,

$$
\sup _{t \geq 0} \mathbb{E}_{0}\left\|U_{0}(t) Y_{0}\right\|_{\alpha,+}^{2}<\infty
$$

Moreover, $\lim _{t \rightarrow \infty} \mathbb{E}_{0}\left|\left\langle U_{0}(t) Y_{0}, \Psi\right\rangle_{+}\right|^{2}=\mathcal{Q}_{\infty}^{\nu}(\Psi, \Psi)$ for any $\Psi \in \mathcal{S}$.

(iii) The limit measure $\nu_{\infty}$ satisfies a mixing condition w.r.t. the group $U_{0}(t)$, i.e., for any

$$
f, g \in L^{2}\left(\mathcal{H}_{\alpha,+}, \nu_{\infty}\right), \lim _{t \rightarrow \infty} \int f\left(U_{0}(t) Y\right) g(Y) \nu_{\infty}(d Y)=\int f(Y) \nu_{\infty}(d Y) \int g(Y) \nu_{\infty}(d Y) .
$$

If $m \neq 0$, then the results of Lemmas 2.6 and Theorem 2.8 follow directly from [5]. In the case $m=0$, the proof of the bound (2.18) needs in some modification (see Appendix C). 


\subsection{Main theorem}

Denote by $\mathcal{Q}_{\infty}(\Psi, \Psi)$ a real quadratic form in $\mathcal{S}$ of the form

$$
\mathcal{Q}_{\infty}(\Psi, \Psi)=\mathcal{Q}_{\infty}^{\nu}\left(\Pi_{\Psi}, \Pi_{\Psi}\right),
$$

where $\mathcal{Q}_{\infty}$ is defined by (2.16), $\Pi_{\Psi}$ is introduced in (4.22).

Remark 2.9 For any $\Psi \in \mathcal{S}, \Pi_{\Psi} \in \mathcal{H}_{1 / \phi,+}$. This follows from 4.22) and Remark 4.5. Therefore, by Remark 2.7 and formula (2.19), $\mathcal{Q}_{\infty}(\Psi, \Psi)$ is bounded for all $\Psi \in \mathcal{S}$.

We define $\mu_{t}$ as the Borel probability measure on $\mathcal{H}_{\alpha,+}$ given the distribution of the solution $Y(t)$ to the problem (1.1)-(1.3), $\mu_{t}(B)=\mu_{0}(U(-t) B)$, where $B \in \mathcal{B}\left(\mathcal{H}_{\alpha,+}\right)$ and $t \in \mathbb{R}$. The main result of the paper is the following theorem.

Theorem 2.10 Let conditions $\mathbf{S 1 - S 4}$ hold and the constants $m, \gamma, \kappa$ satisfy condition $\mathbf{C}$. Then the following assertions are fulfilled.

(i) The measures $\mu_{t}$ weakly converge as $t \rightarrow \infty$ to a limit measure $\mu_{\infty}$ on the space $\mathcal{H}_{\alpha,+}$ with $\alpha<-3 / 2$. Moreover, the limit measure is Gaussian with zero mean value.

(ii) The correlation functions of $\mu_{t}$ converge to a limit, and for $\Psi \in \mathcal{S}$,

$$
\lim _{t \rightarrow \infty} \mathbb{E}\left|\langle Y(t), \Psi\rangle_{+}\right|^{2}=\mathcal{Q}_{\infty}(\Psi, \Psi)
$$

This theorem is proved in Section 5.

Remark 2.11 The limiting energy current at the origin equals $J:=-\gamma \lim _{t \rightarrow \infty} \mathbb{E}(\dot{u}(0, t))^{2}$. Hence, in the case when $\gamma>0, J \neq 0$ if $\int\left(Y^{1}(0)\right)^{2} \mu_{\infty}(d Y) \neq 0$. The limit measures $\mu_{\infty}$ satisfying the last condition can be constructed as follows.

Let the initial correlations functions $q_{0}^{i j}$ from condition S3 have a form $q_{0}^{i j} \equiv 0$ for $i \neq j$, and $q_{0}^{00} \cdot q_{0}^{11} \not \equiv 0$. Write $r(\theta):=\phi^{2}(\theta) \hat{q}_{0}^{00}(\theta)+\hat{q}_{0}^{11}(\theta), \theta \in \mathbb{T}$. Then, $r \in C(\mathbb{T})$ (see Lemma 2.3), $r(\theta) \geq 0$, and $r(\theta) \not \equiv 0$. Moreover, $\hat{q}_{\infty}^{11}(\theta)=r(\theta) / 4$, by (2.15). Using (4.12), we obtain

$$
\int\left(Y^{1}(0)\right)^{2} \mu_{\infty}(d Y)=\lim _{t \rightarrow \infty} \mathbb{E}(\dot{u}(0, t))^{2}=\frac{1}{2 \pi} \int_{\mathbb{T}} \sin ^{2}(\theta) r(\theta)\left|\int_{0}^{+\infty} N(s) e^{i \phi(\theta) s} d s\right|^{2} d \theta \neq 0 .
$$

The properties of $N(t)$ are studied in Appendix B.

\section{Bounds for $q(x, t)$}

In this section, we investigate the behavior of the solutions $q(x, t)$ to the problem (2.5)-(2.8) as $t \rightarrow \infty$. At first, we study the properties of $q(x, t)$ with $x \neq 0$ using the Fourier-Laplace transform.

Definition 3.1 Let $|q(t)| \leq C e^{B t}$. The Fourier-Laplace transform of $q(t)$ is given by the formula

$$
\tilde{q}(\omega)=\int_{0}^{+\infty} e^{i \omega t} q(t) d t, \quad \Im \omega>B
$$


The Gronwall inequality and conditions on $F(q)$ imply standard a priori estimates for the solutions $q(x, t), x \in \mathbb{Z}_{+}$. In particular, there exist constants $A, B<\infty$ such that

$$
\sum_{x \in \mathbb{Z}_{+}}\left(|q(x, t)|^{2}+|\dot{q}(x, t)|^{2}\right) \leq C e^{B t} \quad \text { as } t \rightarrow+\infty
$$

This bound is proved in Appendix A (see formulas (A.17) and (A.18) ). Hence the FourierLaplace transform with respect to $t$-variable, $q(x, t) \rightarrow \tilde{q}(x, \omega)$, exists at least for $\Im \omega>B$ and satisfies the following equation

$$
\left(-\Delta_{L}+m^{2}-\omega^{2}\right) \tilde{q}(x, \omega)=0, \quad x \in \mathbb{N}, \quad \Im \omega>B,
$$

by (2.7). Now we construct the solution of (3.2). We first note that the Fourier transform of the lattice operator $-\Delta_{L}+m^{2}$ is the operator of multiplication by the function $\phi^{2}(\theta)=$ $2-2 \cos \theta+m^{2}$. Thus, $-\Delta_{L}+m^{2}$ is a self-adjoint operator and its spectrum is absolutely continuous and coincides with the range of $\phi^{2}(\theta)$, i.e., with $\left[m^{2}, m^{2}+4\right]$. Second, the following lemma holds (see Lemma 2.1 in [14]).

Lemma 3.2 Denote $\Lambda:=\left[-\sqrt{4+m^{2}},-m\right] \cup\left[m, \sqrt{4+m^{2}}\right]$. For given $\omega \in \mathbb{C} \backslash \Lambda$, the equation

$$
2-2 \cos \theta+m^{2}=\omega^{2}
$$

has the unique solution $\theta(\omega)$ in the domain $\{\theta \in \mathbb{C}: \Im \theta>0,-\pi<\Re \theta \leq \pi\}$. Moreover, $\theta(\omega)$ is an analytic function in $\mathbb{C} \backslash \Lambda$.

Since we seek the solution $q(\cdot, t) \in \ell_{\alpha,+}^{2}$ with some $\alpha, \tilde{q}(x, \omega)$ has a form

$$
\tilde{q}(x, \omega)=\tilde{q}(0, \omega) e^{i \theta(\omega) x}, \quad x \in \mathbb{N} .
$$

We put $\tilde{K}(x, \omega)=e^{i \theta(\omega) x}$. Applying the inverse Fourier-Laplace transform with respect to $\omega$-variable, we write the solution $q(x, t)$ of (2.5) in the form

$$
(q(x, t), \dot{q}(x, t))=\int_{0}^{t} K(x, t-s)(q(0, s), \dot{q}(0, s)) d s, \quad x \in \mathbb{N}, \quad t>0,
$$

where

$$
K(x, t)=\frac{1}{2 \pi} \int_{-\infty+i \mu}^{+\infty+i \mu} e^{-i \omega t} \tilde{K}(x, \omega) d \omega, \quad \tilde{K}(x, \omega)=e^{i \theta(\omega) x}, \quad x \in \mathbb{N}, \quad t>0
$$

with some $\mu>0$. In Appendix A, we study the analytic properties $\tilde{K}(x, \omega)$ for $\omega \in \mathbb{C}$ and obtain the following result.

Theorem 3.3 For any $\alpha<-3 / 2$, the following bound holds,

$$
\|K(\cdot, t)\|_{\alpha,+}^{\prime}:=\sqrt{\sum_{x \in \mathbb{N}}\langle x\rangle^{2 \alpha}|K(x, t)|^{2}} \leq C(1+t)^{-3 / 2} \quad \text { for } \quad t>0 .
$$

In particular,

$$
|K(1, t)| \leq C(1+t)^{-3 / 2}, \quad t>0 .
$$


To estimate $q(0, t)$, we again apply the Fourier-Laplace transform. Using (3.4) and equality $F(q)=-\kappa q$ with $\kappa \geq 0$, we rewrite Eqn (2.6) in the form

$$
\ddot{q}(0, t)=-\left(\kappa+1+m^{2}\right) q(0, t)-\gamma \dot{q}(0, t)+\int_{0}^{t} K(1, t-s) q(0, s) d s+z(1, t) .
$$

At first, we study the solutions of the corresponding homogeneous equation

$$
\ddot{q}(0, t)=-\left(\kappa+1+m^{2}\right) q(0, t)-\gamma \dot{q}(0, t)+\int_{0}^{t} K(1, t-s) q(0, s) d s, \quad t>0,
$$

with the initial data

$$
\left.q(0, t)\right|_{t=0}=u_{0}(0)=: q_{0},\left.\quad \dot{q}(0, t)\right|_{t=0}=v_{0}(0)=: p_{0} .
$$

Applying the Fourier-Laplace transform to the solutions $q(0, t)$ of (3.9) , we obtain

$$
\tilde{D}(\omega) \tilde{q}(0, \omega)=-i \omega q_{0}+q_{0} \gamma+p_{0} \quad \text { for } \Im \omega>B,
$$

where, by definition,

$$
\tilde{D}(\omega):=-\omega^{2}+\kappa+1+m^{2}-i \omega \gamma-\tilde{K}(1, \omega), \quad \tilde{K}(1, \omega)=e^{i \theta(\omega)} .
$$

Write $\tilde{N}(\omega):=[\tilde{D}(\omega)]^{-1}$. Then $\tilde{q}(0, \omega)=\tilde{N}(\omega)\left(-i \omega q_{0}+q_{0} \gamma+p_{0}\right)$. The analytic properties of $\tilde{D}(\omega)$ and $\tilde{N}(\omega)$ for $\omega \in \mathbb{C}$ are studied in Appendix B. In particular, we prove that $\tilde{N}(\omega)$ is analytic in the upper half-space, i.e., with $\Im \omega>0$. Denote

$$
N(t)=\frac{1}{2 \pi} \int_{-\infty+i \mu}^{+\infty+i \mu} e^{-i \omega t} \tilde{N}(\omega) d \omega, \quad t \geq 0, \quad \text { with some } \mu>0 .
$$

The following theorem is proved in Appendix B.

Theorem 3.4 Let constants $m, \gamma, \kappa$ be nonnegative and satisfy condition $\mathbf{C}$. Then

$$
\left|N^{(k)}(t)\right| \leq C(1+t)^{-3 / 2}, \quad t \geq 0, \quad k=0,1,2 .
$$

If condition $\mathbf{C}$ is not satisfied, then $N(t)$ decreases more slowly than $\langle t\rangle^{-3 / 2}$, see Remark B.8. We need the bound (3.13) to derive asymptotics (4.3) which plays the crucial role in our proof.

Corollary 3.5 Denote by $S(t)$ a solving operator of the Cauchy problem (3.9), (3.10). Then the variation constants formula gives the following representation for the solution of the problem (3.8), (3.10):

$$
\left(\begin{array}{c}
q(0, t) \\
\dot{q}(0, t)
\end{array}\right)=S(t)\left(\begin{array}{c}
q_{0} \\
p_{0}
\end{array}\right)+\int_{0}^{t} S(\tau)\left(\begin{array}{c}
0 \\
z(1, t-\tau)
\end{array}\right) d \tau, \quad t>0
$$

Evidently, $S(0)=I$. Moreover, the matrix $S(t)$ has a form $\left(\begin{array}{cc}\dot{N}(t)+\gamma N(t) & N(t) \\ \ddot{N}(t)+\gamma \dot{N}(t) & \dot{N}(t)\end{array}\right)$. By Theorem 3.4. $|S(t)| \leq C(1+t)^{-3 / 2}$, and the solutions of (3.8) satisfy the following bound:

$$
|q(0, t)|+|\dot{q}(0, t)| \leq C_{1}(1+t)^{-3 / 2}\left(\left|q_{0}\right|+\left|p_{0}\right|\right)+C_{2} \int_{0}^{t}(1+s)^{-3 / 2}|z(1, t-s)| d s, \quad t \geq 0 .
$$




\section{Asymptotic behavior of $Y(t)$ as $t \rightarrow \infty$ in mean}

Set $q^{(0)}(x, t)=q(x, t), q^{(1)}(x, t)=\dot{q}(x, t), x \in \mathbb{Z}_{+}$.

\subsection{The estimates of $q(0, t)$ in mean}

To derive the asymptotic behavior for $q^{(j)}(0, t)$ in mean, introduce the following notations. Write (see (2.11) $)$

$$
\mathbf{G}_{z}^{i}(y, t):=\left(\mathcal{G}_{t,+}^{i 0}(z, y), \mathcal{G}_{t,+}^{i 1}(z, y)\right)=\left(\mathcal{G}_{t}^{i 0}(z-y)-\mathcal{G}_{t}^{i 0}(z+y), \mathcal{G}_{t}^{i 1}(z-y)-\mathcal{G}_{t}^{i 1}(z+y)\right)
$$

$y, z \in \mathbb{Z}, i=0,1, t \in \mathbb{R}$. Let $\mathbf{G}^{j}(y)$ denote the vector valued functions defined as

$$
\mathbf{G}^{j}(y)=\int_{0}^{+\infty} N(s) \mathbf{G}_{1}^{j}(y,-s) d s=\int_{0}^{+\infty} N^{(j)}(s) \mathbf{G}_{1}^{0}(y,-s) d s, \quad y \in \mathbb{Z}, \quad j=0,1,
$$

where the function $N(s)$ is introduced in (3.12), $\mathbf{G}_{1}^{j}(y, s)$ is defined in (4.1).

Proposition 4.1 Let conditions $\mathbf{S 1 - S 4}$ and $\mathbf{C}$ hold, $Y_{0} \in \mathcal{H}_{\alpha,+}$, and $q(0, t)$ be a solution of the problem (3.8), (3.10). Then

$$
q^{(j)}(0, t)=\left\langle U_{0}(t) Y_{0}, \mathbf{G}^{j}\right\rangle_{+}+r_{j}(t), \quad t>0, \quad j=0,1, \quad \text { where } \mathbb{E}\left|r_{j}(t)\right|^{2} \leq C(1+t)^{-1},
$$

the vector valued functions $\mathbf{G}^{j}$ are defined in (4.2).

Proof First, Corollary 3.5 and the bound (3.13) imply that

$$
\mathbb{E}\left|\left(\begin{array}{c}
q(0, t) \\
\dot{q}(0, t)
\end{array}\right)-\int_{0}^{t} S(\tau)\left(\begin{array}{c}
0 \\
z(1, t-\tau)
\end{array}\right) d \tau\right|^{2} \leq C(1+t)^{-3}, \quad t>0 .
$$

Second, $S(\tau)\left(\begin{array}{c}0 \\ z(1, t-\tau)\end{array}\right)=\left(\begin{array}{c}N(\tau) \\ \dot{N}(\tau)\end{array}\right) z(1, t-\tau)$. Moreover, for $j=0,1$,

$$
\mathbb{E}\left|\int_{t}^{+\infty} N^{(j)}(\tau) z(1, t-\tau) d \tau\right|^{2}=\int_{t}^{+\infty} N^{(j)}\left(\tau_{1}\right) d \tau_{1} \int_{t}^{+\infty} N^{(j)}\left(\tau_{2}\right) \mathbb{E}\left(z\left(1, t-\tau_{1}\right) z\left(1, t-\tau_{2}\right)\right) d \tau_{2} .
$$

Further, the bound (2.18) gives

$$
\left|\mathbb{E}\left(z\left(1, t-\tau_{1}\right) z\left(1, t-\tau_{2}\right)\right)\right| \leq C \sup _{s \in \mathbb{R}} \mathbb{E}|z(1, s)|^{2} \leq C_{1}<\infty .
$$

Hence, the bound (3.13) yields

$$
\mathbb{E}\left|\int_{t}^{+\infty} S(\tau)\left(\begin{array}{c}
0 \\
z(1, t-\tau)
\end{array}\right) d \tau\right|^{2} \leq C \sup _{s \in \mathbb{R}} \mathbb{E}|z(1, s)|^{2}\left(\int_{t}^{+\infty}(1+\tau)^{-3 / 2} d \tau\right)^{2} \leq C_{1}(1+t)^{-1} .
$$

This implies the representation (4.3), since by (4.1) and (2.10),

$$
z(1, t-\tau)=\left\langle U_{0}(t) Y_{0}(\cdot), \mathbf{G}_{1}^{0}(\cdot,-\tau)\right\rangle_{+} \cdot
$$


Remark 4.2 (i) By (2.11) and (2.12), $\mathbf{G}_{z}^{i}(y, t)$ is odd w.r.t. $y \in \mathbb{Z}$. Also, the Parseval identity gives

$$
\left\|\mathbf{G}_{z}^{i}(\cdot, t)\right\|_{0}^{2}=\frac{1}{2 \pi} \int_{-\pi}^{\pi}\left|\hat{\mathbf{G}}_{z}^{i}(\theta, t)\right|^{2} d \theta=\frac{2}{\pi} \int_{-\pi}^{\pi}\left(\left|\hat{\mathcal{G}}_{t}^{i 0}(\theta)\right|^{2}+\left|\hat{\mathcal{G}}_{t}^{i 1}(\theta)\right|^{2}\right) \sin ^{2}(z \theta) d \theta, \quad z \in \mathbb{Z} .
$$

Hence, if $m \neq 0$, then $\left\|\mathbf{G}_{z}^{i}(\cdot, t)\right\|_{0}^{2} \leq C<\infty$ uniformly in $z \in \mathbb{Z}$ and $t \in \mathbb{R}$. If $m=0$, then $\left\|\mathbf{G}_{z}^{1}(\cdot, t)\right\|_{0}^{2} \leq C<\infty$ uniformly on $z$ and $t$, and

$$
\left\|\mathbf{G}_{z}^{0}(\cdot, t)\right\|_{0}^{2} \leq C_{1}+C_{2} \int_{\pi}^{\pi} \frac{\sin ^{2}(z \theta)}{\sin ^{2}(\theta / 2)} d \theta \leq C_{1}+C_{2}|z|, \quad z \in \mathbb{Z} .
$$

In particular, $\hat{\mathbf{G}}_{1}^{0}(\theta, t)=2 i \sin \theta(\cos (\phi(\theta) t), \sin (\phi(\theta) t) / \phi(\theta)), \theta \in \mathbb{T}$. Hence,

$$
\left\|\mathbf{G}_{1}^{0}(\cdot, t)\right\|_{0}^{2}=C \int_{-\pi}^{\pi}\left(\cos ^{2}(\phi(\theta) t)+\frac{\sin ^{2}(\phi(\theta) t)}{\phi^{2}(\theta)}\right) \sin ^{2}(\theta) d \theta \leq C<\infty .
$$

(ii) $\mathbf{G}^{j}(\cdot)$ is odd. Moreover, by the bounds (3.13) and (4.5), $\mathbf{G}^{j} \in \mathcal{H}_{0}$, since

$$
\left\|\mathbf{G}^{j}(\cdot)\right\|_{0} \leq \int_{0}^{+\infty}\left|N^{(j)}(s)\right|\left\|\mathbf{G}_{1}^{0}(\cdot,-s)\right\|_{0} d s \leq C \int_{0}^{+\infty}\left|N^{(j)}(s)\right| d s<\infty .
$$

In Fourier transform,

$$
\hat{\mathbf{G}}^{j}(\theta)=2 i \sin \theta \int_{0}^{+\infty} N^{(j)}(s)(\cos (\phi(\theta) s),-\sin (\phi(\theta) s) / \phi(\theta)) d s, \quad \theta \in \mathbb{T} .
$$

Therefore, $\mathbf{G}^{j} \in \mathcal{H}_{1 / \phi}:=\ell_{1 / \phi}^{2} \times \ell^{2}$, where $\ell_{1 / \phi}^{2}=\left\{\psi \in \ell^{2}: \hat{\psi} / \phi \in L^{2}(\mathbb{T})\right\}$. Note that $\ell_{1 / \phi}^{2} \equiv \ell^{2}$ if $m \neq 0$.

Denote by $U_{0}^{\prime}(t)$ the operator adjoint to $U_{0}(t)$ :

$$
\left\langle Y, U_{0}^{\prime}(t) \Psi\right\rangle_{+}=\left\langle U_{0}(t) Y, \Psi\right\rangle_{+}, \quad Y \in \mathcal{H}_{\alpha,+}, \quad \Psi \in \mathcal{S}, \quad t \in \mathbb{R} .
$$

In other words,

$$
\left(U_{0}^{\prime}(t) \Psi\right)^{j}(y)=\sum_{i=0,1} \sum_{x \in \mathbb{Z}_{+}} \mathcal{G}_{t,+}^{i j}(x, y) \Psi^{i}(x) \quad \text { for } \quad \Psi=\left(\Psi^{0}, \Psi^{1}\right) \in \mathcal{S}, \quad t \in \mathbb{R}, \quad y \in \mathbb{Z}_{+}, \quad j=0,1 .
$$

In particular, $\mathbf{G}_{1}^{0}(y, t)=\left(U_{0}^{\prime}(t) \Psi\right)(y)$ with $\Psi(x)=\left(\delta_{1 x}, 0\right)$ (see (4.1)), where $\delta_{1 x}$ denotes the Kronekker symbol.

Corollary 4.3 (i) For $\mathbf{G}^{j}$ defined in (4.2), we have

$$
\sup _{t \in \mathbb{R}} \mathbb{E}\left|\left\langle U_{0}(t) Y_{0}, \mathbf{G}^{j}\right\rangle_{+}\right|^{2}=\sup _{t \in \mathbb{R}} \mathbb{E}\left|\left\langle Y_{0}, U_{0}^{\prime}(t) \mathbf{G}^{j}\right\rangle_{+}\right|^{2} \leq C<\infty .
$$

Indeed, since $U_{0}^{\prime}(t) \mathbf{G}_{1}^{0}(y, \tau)=\mathbf{G}_{1}^{0}(y, \tau+t)$, we have

$$
\sup _{t \in \mathbb{R}}\left\|U_{0}^{\prime}(t) \mathbf{G}^{j}\right\|_{0} \leq C<\infty
$$


by (4.2), (4.5) and (3.13). Hence, (4.9) follows from the bounds (2.9) and (4.10).

(ii) The representation (4.3), Lemma 2.6 and the bound (4.9) give the following convergence,

$$
\mathbb{E}\left(q^{(i)}(0, t) q^{(j)}(0, t)\right) \rightarrow \mathcal{Q}_{\infty}^{\nu}\left(\mathbf{G}^{i}, \mathbf{G}^{j}\right) \quad \text { as } t \rightarrow \infty,
$$

where the quadratic form $\mathcal{Q}_{\infty}^{\nu}$ is defined by (2.16). The r.h.s. of (4.11) is defined by Remarks 2.7 and 4.2 (ii). Furthermore, using formulas (2.17), (4.7), and (2.15), we obtain $\mathcal{Q}_{\infty}^{\nu}\left(\mathbf{G}^{i}, \mathbf{G}^{j}\right)=0$ for $i \neq j, i, j=0,1$, and

$$
\lim _{t \rightarrow \infty} \mathbb{E}\left(q^{(i)}(0, t)\right)^{2}=\mathcal{Q}_{\infty}^{\nu}\left(\mathbf{G}^{i}, \mathbf{G}^{i}\right)=\frac{2}{\pi} \int_{\mathbb{T}} \sin ^{2}(\theta) \hat{q}_{\infty}^{i i}(\theta)\left|\int_{0}^{+\infty} N(s) e^{i \phi(\theta) s} d s\right|^{2} d \theta .
$$

\subsection{The large time behavior of $q(x, t), x \in \mathbb{N}$, in mean}

Let $\mathbf{K}^{j}(x, y) \quad j=0,1, x \in \mathbb{N}, y \in \mathbb{Z}$, stand for vector-valued functions of a form

$$
\mathbf{K}^{j}(x, y)=\int_{0}^{+\infty} K(x, s)\left(U_{0}^{\prime}(-s) \mathbf{G}^{j}\right)(y) d s=\int_{0}^{+\infty} \int_{0}^{+\infty} K(x, s) N^{(j)}(\tau) \mathbf{G}_{1}^{0}(y,-s-\tau) d s d \tau
$$

where $K(x, s)$ is defined in (3.5), $\mathbf{G}^{j}$ is introduced in (4.2). Note that $\mathbf{K}^{j}(x, y)$ is odd w.r.t. $y \in \mathbb{Z}, \mathbf{K}^{j}(x, \cdot) \in \mathcal{H}_{0}$ for any $x \in \mathbb{N}$ and $\left\|\mathbf{K}^{j}(x, \cdot)\right\|_{0} \in \mathcal{H}_{\alpha,+}$ with $\alpha<-3 / 2$ by (3.6) and (4.10).

Lemma 4.4 Assume that $\alpha<-3 / 2$. Then the following assertions are true.

(i) The solution $q(x, t), x \in \mathbb{N}$, of the problem (2.5)-(2.8) admits the following representation

$$
q^{(j)}(x, t)=\left\langle U_{0}(t) Y_{0}, \mathbf{K}^{j}(x, \cdot)\right\rangle_{+}+r_{j}(x, t), \quad j=0,1, \quad t>0,
$$

where $\mathbb{E}\left(\left\|r_{j}(\cdot, t)\right\|_{\alpha,+}^{\prime}\right)^{2} \leq C\langle t\rangle^{-1}$, and the notation $\|\cdot\|_{\alpha,+}^{\prime}$ is introduced in (3.6).

(ii) The correlation functions converge to a limit,

$$
\lim _{t \rightarrow \infty} \mathbb{E}\left(q^{(i)}(x, t) q^{(j)}\left(x^{\prime}, t\right)\right)=\mathcal{Q}_{\infty}^{\nu}\left(\mathbf{K}^{i}(x, \cdot), \mathbf{K}^{j}\left(x^{\prime}, \cdot\right)\right) \quad \text { for any } x, x^{\prime} \in \mathbb{Z}_{+}, \quad i, j=0,1 .
$$

Proof At first, by (3.4) and (4.3),

$$
q^{(j)}(x, t)=\int_{0}^{t} K(x, t-s) q^{(j)}(0, s) d s=\int_{0}^{t} K(x, t-s)\left\langle U_{0}(s) Y_{0}, \mathbf{G}^{j}(\cdot)\right\rangle_{+} d s+r_{j}^{\prime}(x, t),
$$

where $x \in \mathbb{N}, \mathbb{E}\left\|r_{j}^{\prime}(\cdot, t)\right\|_{\alpha,+}^{2} \leq C\langle t\rangle^{-1}$. Indeed, by (4.3) and (3.6),

$$
\begin{aligned}
\mathbb{E}\left(\left\|r_{j}^{\prime}(\cdot, t)\right\|_{\alpha,+}^{\prime}\right)^{2} & =\mathbb{E}\left(\left\|\int_{0}^{t} K(\cdot, t-s) r_{j}(s) d s\right\|_{\alpha,+}^{\prime}\right)^{2} \leq\left(\int_{0}^{t}\|K(\cdot, t-s)\|_{\alpha,+}^{\prime} \sqrt{\mathbb{E}\left|r_{j}(s)\right|^{2}} d s\right)^{2} \\
& \leq C\left(\int_{0}^{t}(1+t-s)^{-3 / 2}(1+s)^{-1 / 2} d s\right)^{2} \leq C_{1}\langle t\rangle^{-1} .
\end{aligned}
$$

Second, the first term in the r.h.s. of (4.15) has a form (see (4.13))

$$
\int_{0}^{t} K(x, s)\left\langle U_{0}(t-s) Y_{0}, \mathbf{G}^{j}(\cdot)\right\rangle_{+} d s=\left\langle U_{0}(t) Y_{0}, \mathbf{K}^{j}(x, \cdot)\right\rangle_{+}+r_{j}^{\prime \prime}(x, t),
$$


where, by definition,

$$
r_{j}^{\prime \prime}(x, t)=\int_{t}^{+\infty} K(x, s)\left\langle U_{0}(t-s) Y_{0}, \mathbf{G}^{j}\right\rangle_{+} d s .
$$

The bounds (3.6) and (4.9) yield

$$
\mathbb{E}\left(\left\|r_{j}^{\prime \prime}(\cdot, t)\right\|_{\alpha,+}^{\prime}\right)^{2} \leq\left(\int_{t}^{+\infty}\|K(\cdot, s)\|_{\alpha,+}^{\prime}\left(\mathbb{E}_{0}\left|\left\langle U_{0}(t-s) Y_{0}, \mathbf{G}^{j}\right\rangle_{+}\right|^{2}\right)^{1 / 2} d s\right)^{2} \leq C\langle t\rangle^{-1}
$$

Hence, the bounds (4.15)-(4.17) imply (4.14) with $r_{j}(x, t)=r_{j}^{\prime}(x, t)+r_{j}^{\prime \prime}(x, t)$. Finally, the assertion (ii) of the lemma follows from the representation (4.14) and Lemma 2.6.

Remark Set $\tilde{K}(0, \omega):=\left.e^{i \theta(\omega) x}\right|_{x=0}=1$. Then, formally, $K(0, t)=\delta_{0 t}$. Hence, we can put $\mathbf{K}^{j}(0, y)=\mathbf{G}^{j}(y), y \in \mathbb{Z}$. Then the representation (4.3) follows from (4.14).

For any $\psi \in S$ and $j=0,1$, denote

$$
\mathbf{K}_{\psi}^{j}(y):=\left\langle\mathbf{K}^{j}(\cdot, y), \psi(\cdot)\right\rangle_{+}=\int_{0}^{+\infty}\langle K(\cdot, s), \psi\rangle_{+}\left(U_{0}^{\prime}(-s) \mathbf{G}^{j}\right)(y) d s, \quad y \in \mathbb{Z} .
$$

Remark 4.5 By Remark 4.2 and (4.13), $\mathbf{K}^{j}(x, y)$ is odd w.r.t. $y \in \mathbb{Z}$. Then, $\mathbf{K}_{\psi}^{j}$ is odd. Moreover, $\mathbf{K}^{j}(x, \cdot) \in \mathcal{H}_{1 / \phi} \equiv \ell_{1 / \phi}^{2} \times \ell^{2}$ for any $x \in \mathbb{Z}_{+}$. Therefore, $\mathbf{K}_{\psi}^{j} \in \mathcal{H}_{1 / \phi}$.

Corollary 4.6 (i) For any $\psi \in S$ and $j=0,1$,

$$
\left\langle q^{(j)}(\cdot, t), \psi\right\rangle_{+}=\left\langle U_{0}(t) Y_{0}, \mathbf{K}_{\psi}^{j}\right\rangle_{+}+r_{j}(t), \quad t>0, \quad \text { where } \mathbb{E}\left|r_{j}(t)\right|^{2} \leq C\langle t\rangle^{-1} .
$$

(ii) For any $\psi \in S$,

$$
\sup _{t \in \mathbb{R}} \mathbb{E}\left|\left\langle U_{0}(t) Y_{0}, \mathbf{K}_{\psi}^{j}\right\rangle_{+}\right|^{2}=\sup _{t \in \mathbb{R}} \mathbb{E}\left|\left\langle Y_{0}, U_{0}^{\prime}(t) \mathbf{K}_{\psi}^{j}\right\rangle_{+}\right|^{2} \leq C<\infty .
$$

(iii) For any $\psi, \chi \in S, \mathbb{E}\left(\left\langle q^{(i)}(\cdot, t), \psi\right\rangle_{+}\left\langle q^{(j)}(\cdot, t), \chi\right\rangle_{+}\right) \rightarrow \mathcal{Q}_{\infty}^{\nu}\left(\mathbf{K}_{\psi}^{i}, \mathbf{K}_{\chi}^{j}\right)$ as $t \rightarrow \infty$.

Proof The representation (4.19) follows from (4.14). Now we verify (4.20). By (4.18),

$$
U_{0}^{\prime}(t) \mathbf{K}_{\psi}^{j}=\int_{0}^{+\infty}\langle K(\cdot, s), \psi\rangle_{+} U_{0}^{\prime}(t-s) \mathbf{G}^{j} d s .
$$

Hence, (4.10) and (3.6) imply the following uniform bound,

$$
\sup _{t \in \mathbb{R}}\left\|U_{0}^{\prime}(t) \mathbf{K}_{\psi}^{j}\right\|_{0} \leq C \int_{0}^{+\infty}\|K(\cdot, s)\|_{\alpha,+} d s<\infty .
$$

Therefore, (4.20) follows from the bounds (2.9) and (4.21). Finally, (4.19) and Lemma 2.6 imply the part (iii) of Corollary 4.6.

Write $\Pi^{j}(x, y)=e_{j} \delta_{x y}+\mathbf{K}^{j}(x, y)$ with $e_{0}=(1,0), e_{1}=(0,1), j=0,1, x, y \in \mathbb{Z}_{+}$. Given $\Psi=\left(\Psi^{0}, \Psi^{1}\right) \in \mathcal{S}$, define the vector valued functions $\Pi_{\Psi}(y), y \in \mathbb{Z}_{+}$,

$$
\Pi_{\Psi}(y):=\sum_{j=0}^{1}\left\langle\Pi^{j}(\cdot, y), \Psi^{j}\right\rangle_{+}=\Psi(y)+\sum_{j=0}^{1}\left\langle\mathbf{K}^{j}(\cdot, y), \Psi^{j}(\cdot)\right\rangle_{+}=\Psi(y)+\sum_{j=0}^{1} \mathbf{K}_{\Psi^{j}}^{j}(y)
$$

with $\mathbf{K}_{\psi}^{j}(y)$ from (4.18). The formula (2.1) and Lemma 4.4 imply the following lemma. 
Lemma 4.7 For the solution $Y(t)=(u(x, t), \dot{u}(x, t)) \equiv\left(Y^{0}(x, t), Y^{1}(x, t)\right)$ of the problem (1.1)-(1.3), the following asymptotics holds,

$$
Y^{j}(x, t)=\left\langle U_{0}(t) Y_{0}, \Pi^{j}(x, \cdot)\right\rangle_{+}+r_{j}(x, t), \quad j=0,1,
$$

where $r_{j}(x, t)$ is introduced in (4.14) and $\mathbb{E}\left\|r_{j}(\cdot, t)\right\|_{\alpha,+}^{2} \leq C\langle t\rangle^{-1}(\alpha<-3 / 2)$. Hence, for any $\Psi=\left(\Psi^{0}, \Psi^{1}\right) \in \mathcal{S}$,

$$
\langle Y(t), \Psi\rangle_{+}=\left\langle U_{0}(t) Y_{0}, \Pi_{\Psi}\right\rangle_{+}+r(t)
$$

where $r(t)=\sum_{j=0}^{1}\left\langle r_{j}(\cdot, t), \Psi^{j}\right\rangle_{+}$and $\mathbb{E}|r(t)|^{2} \leq C\langle t\rangle^{-1}$.

\section{Proof of Theorem 2.10}

The compactness of the measures family $\left\{\mu_{t}, t>0\right\}$ follows from the Prokhorov compactness theorem and the bound (5.1) below, since the embedding $\mathcal{H}_{\alpha,+} \subset \mathcal{H}_{\beta,+}$ is compact if $\alpha>\beta$.

Lemma 5.1 Let $\alpha<-3 / 2$ and conditions $\mathbf{S 1 - S 4}$ and $\mathbf{C}$ hold. Then

$$
\sup _{t \geq 0} \mathbb{E}\left\|U(t) Y_{0}\right\|_{\alpha,+}^{2} \leq C<\infty
$$

Proof By (2.1), the solution $Y(\cdot, t)=U(t) Y_{0}$ has a form $Y(x, t)=Z(x, t)+X(x, t)$, where $Z(x, t)=U_{0}(t) Y_{0}$ and $X(x, t)=(q(x, t), \dot{q}(x, t))$. Hence, by the bound (2.18), to prove the bound (5.1) it suffices to verify that

$$
\sup _{t \geq 0} \mathbb{E}\|X(\cdot, t)\|_{\alpha,+}^{2} \leq C<\infty .
$$

Applying (3.4) and (3.6), we have

$$
\begin{aligned}
\mathbb{E}\|X(\cdot, t)\|_{\alpha,+}^{2} & =\mathbb{E}|X(0, t)|^{2}+\mathbb{E} \sum_{x \in \mathbb{N}}\langle x\rangle^{2 \alpha}\left|\int_{0}^{t} K(x, t-s) X(0, s) d s\right|^{2} \\
& \leq C \sup _{\tau \in[0, t]} \mathbb{E}|X(0, \tau)|^{2}
\end{aligned}
$$

Using the estimate (3.14), we obtain

$$
\mathbb{E}|X(0, t)|^{2} \leq \mathbb{E}\left(\left|q_{0}\right|^{2}+\left|p_{0}\right|^{2}\right)+C \sup _{\tau \geq 0} \mathbb{E}|z(1, \tau)|^{2}, \quad t \geq 0 .
$$

On the other hand, $\sup _{\tau \in \mathbb{R}} \mathbb{E}|z(1, \tau)|^{2}=\sup _{\tau \in \mathbb{R}} Q_{\tau}^{00}(1,1) \leq C<\infty$ by (2.18). Hence,

$$
\sup _{\tau \geq 0} \mathbb{E}|X(0, t)|^{2} \leq C<\infty .
$$

Estimates (5.3) and (5.4) imply the bound (5.2).

To end the proof of assertion (i) of Theorem 2.10, it suffices to check the convergence (1.7) of characteristic functionals for $\mu_{t}$. By the triangle inequality and the equality (2.19),

$$
\begin{aligned}
\left|\mathbb{E} e^{i\langle Y(t), \Psi\rangle_{+}}-e^{-\frac{1}{2} \mathcal{Q}_{\infty}(\Psi, \Psi)}\right| \leq & \left|\mathbb{E}\left(e^{i\langle Y(t), \Psi\rangle_{+}}-e^{i\left\langle U_{0}(t) Y_{0}, \Pi_{\Psi}\right\rangle_{+}}\right)\right| \\
& +\left|\mathbb{E}_{0}\left(e^{i\left\langle U_{0}(t) Y_{0}, \Pi_{\Psi}\right\rangle_{+}}\right)-e^{-\frac{1}{2} \mathcal{Q}_{\infty}^{\nu}\left(\Pi_{\Psi}, \Pi_{\Psi}\right)}\right|
\end{aligned}
$$


Applying (4.23), we estimate the first term in the r.h.s. of (5.5) by

$$
\mathbb{E}\left|\langle Y(t), \Psi\rangle_{+}-\left\langle U_{0}(t) Y_{0}, \Pi_{\Psi}\right\rangle_{+}\right| \leq \mathbb{E}|r(t)| \leq\left(\mathbb{E}|r(t)|^{2}\right)^{1 / 2} \leq C\langle t\rangle^{-1 / 2} \rightarrow 0 \quad \text { as } t \rightarrow \infty .
$$

The convergence of the characteristic functionals $\hat{\nu}_{t}\left(\Pi_{\Psi}\right)=\mathbb{E}_{0}\left(\exp \left\{i\left\langle U_{0}(t) Y_{0}, \Pi_{\Psi}\right\rangle_{+}\right\}\right)$to a limit as $t \rightarrow \infty$ follows from Theorem 2.8 and Remark 2.9. This proves the convergence (1.7). Corollary 4.6 and Lemma 2.6 imply assertion (ii) of Theorem 2.10.

\section{ACKNOWLEDGMENTS}

This work was supported partly by the research grant of RFBR (Grant No. 15-01-03587). The author is grateful to Herbert Spohn for the statement of the problem and to Alexander Komech for useful discussions concerning several aspects of this paper.

\section{Appendix A: The behavior of $K(x, t)$ as $t \rightarrow \infty$}

In this section, we first study the properties of $\tilde{K}(x, \omega):=e^{i \theta(\omega) x}$ (the function $\theta(\omega)$ is introduced in Lemma 3.2) applying the technique of [14, 6]. Next, using these properties, we obtain the bound (3.6) for $K(x, t)$. Finally, by the bound (3.6), we prove the existence of solutions of the problem (1.1)-(1.3).

\section{A.1 Properties of $e^{i \theta(\omega) x}$ for $\omega \in \mathbb{C}$ and $x \in \mathbb{N}$}

We set $\Lambda:=\left[-\sqrt{4+m^{2}},-m\right] \cup\left[m, \sqrt{4+m^{2}}\right]$ and $\Lambda_{0}=\left\{ \pm m, \pm \sqrt{4+m^{2}}\right\}$. We indicate the properties (I)-(III) of the function $\tilde{K}(x, \omega)$ for different values of $\omega$ such as $\omega \in \mathbb{C} \backslash \Lambda$, $\omega \in \Lambda \backslash \Lambda_{0}$, and $\omega \in \Lambda_{0}$.

(I) Let $\omega \in \mathbb{C} \backslash \Lambda$. Then $\Im \theta(\omega)>0$. In this case, $\tilde{K}(x, \omega)$ exponentially decays in $x$. Hence, $\tilde{K}(x, \omega)$ is an analytic function in the complex $\omega$-plane with the values in the class $\ell_{\alpha,+}^{2}$. Moreover, by (3.3) and the condition $\Im \theta(\omega)>0$, we have

$$
\left|e^{i \theta(\omega)}\right| \leq C|\omega|^{-2} \quad \text { as }|\omega| \rightarrow \infty
$$

Remark A.1 It follows from (3.5) and (A.1) that there exist constants $C, B<\infty$ such that

$$
\sum_{x \in \mathbb{N}}\langle x\rangle^{2 \alpha}|K(x, t)|^{2} \leq C e^{B t} \quad \text { for any } \alpha \in \mathbb{R}, \quad t>0 .
$$

(II) Let $\omega \in \Lambda \backslash \Lambda_{0}$. Then we have the pointwise convergence $\tilde{K}(x, \omega \pm i \varepsilon) \rightarrow \tilde{K}(x, \omega \pm i 0)$ as $\varepsilon \rightarrow+0$ for any $x \in \mathbb{N}$. Moreover, $|\tilde{K}(x, \omega \pm i \varepsilon)| \leq C$ uniformly in $\varepsilon$. Hence, for any $\alpha<-1 / 2$ and $\omega \notin \Lambda_{0}$,

$$
\sum_{x \in \mathbb{N}}\langle x\rangle^{2 \alpha}|\tilde{K}(x, \omega \pm i 0)-\tilde{K}(x, \omega \pm i \varepsilon)|^{2} \rightarrow 0 \quad \text { as } \varepsilon \rightarrow+0
$$

by the Lebesgue dominated convergence theorem. Furthermore, since $\overline{\theta(\omega)}=-\theta(\bar{\omega})$ for $\omega \in \mathbb{C} \backslash \Lambda, \tilde{K}(x, \omega-i 0)=\overline{\tilde{K}(x, \omega+i 0)}$ for $\omega \in \Lambda \backslash \Lambda_{0}$ and $x \in \mathbb{N}$. 
(III) Now we study the behavior of $\tilde{K}(x, \omega)$ near the points $\omega \in \Lambda_{0}=\left\{ \pm m, \pm \sqrt{4+m^{2}}\right\}$. Eqn (3.3) implies

$$
e^{i \theta(\omega)}=\cos \theta(\omega)+i \sin \theta(\omega)=1-\frac{1}{2}\left(\omega^{2}-m^{2}\right)+\frac{i}{2} \sqrt{\left(\omega^{2}-m^{2}\right)\left(4+m^{2}-\omega^{2}\right)}, \quad \omega \in \mathbb{C} \backslash \Lambda .
$$

The Taylor expansion implies

$$
e^{i \theta(\omega)}=1+i \sqrt{\omega^{2}-m^{2}}-\frac{1}{2}\left(\omega^{2}-m^{2}\right)-\frac{i}{8}\left(\omega^{2}-m^{2}\right)^{3 / 2}+\ldots, \quad \omega \rightarrow \pm m,
$$

where $\omega \in \mathbb{C}_{+}:=\{\omega \in \mathbb{C}: \Im \omega>0\}, \Im \sqrt{\omega^{2}-m^{2}}>0$. Here $\operatorname{sgn}\left(\Re \sqrt{\omega^{2}-m^{2}}\right)=\operatorname{sgn}(\Re \omega)$ for $\omega \in \mathbb{C}_{+}$. This choice of the branch of the complex root $\sqrt{\omega^{2}-m^{2}}$ follows from the condition $\Im \theta(\omega)>0$. Hence, for $x \in \mathbb{N}$,

$$
e^{i \theta(\omega) x}=1+\sum_{j=1}^{+\infty}\left(\omega^{2}-m^{2}\right)^{j / 2} R^{j}(x), \quad \omega \rightarrow \pm m+i 0 .
$$

Here polynomials $R^{j}(x)$ are of a form $R^{j}(x)=\sum_{k=1}^{j} c_{k}^{j} x^{k}$ with coefficients $c_{k}^{j} \in \mathbb{C}, j \in \mathbb{N}$. For example, $R^{1}(x)=i x, R^{2}(x)=-x^{2} / 2, R^{3}(x)=-i\left(4 x^{3}-x\right) / 24$.

Similarly,

$$
e^{i \theta(\omega)}=-1+i \sqrt{m^{2}+4-\omega^{2}}+\frac{1}{2}\left(m^{2}+4-\omega^{2}\right)-\frac{i}{8}\left(m^{2}+4-\omega^{2}\right)^{3 / 2}+\ldots
$$

as $\omega \rightarrow \pm \sqrt{m^{2}+4}, \omega \in \mathbb{C}_{+}$. Here $\sqrt{m^{2}+4-\omega^{2}}$ is the complex root and we choose the branch of this root so that $\operatorname{sgn}\left(\Re \sqrt{m^{2}+4-\omega^{2}}\right)=\operatorname{sgn}(\Re \omega)$, which follows from the condition $\Im \theta(\omega)>0$. Hence, for $x \in \mathbb{N}$,

$$
e^{i \theta(\omega) x}=(-1)^{x}\left(1-i x \sqrt{m^{2}+4-\omega^{2}}-\left(m^{2}+4-\omega^{2}\right) x^{2} / 2+\ldots\right)
$$

as $\omega \rightarrow \pm \sqrt{m^{2}+4}+i 0$. If $m=0$, then (A.3) and the Taylor expansion give

$$
e^{i \theta(\omega)}=1-\frac{\omega^{2}}{2}+i \omega\left(1-\frac{\omega^{2}}{8}-\frac{\omega^{4}}{128}+\ldots\right) \quad \text { as } \omega \rightarrow 0,
$$

and $e^{i \theta(\omega)}=-1+i \sqrt{4-\omega^{2}}+\ldots$ as $\omega \rightarrow \pm 2, \omega \in \mathbb{C}_{+}$. Therefore, in the case $m=0$,

$$
e^{i \theta(\omega) x}=\left\{\begin{array}{ll}
1+i \omega x-\omega^{2} x^{2} / 2-i \omega^{3}\left(4 x^{3}-x\right) / 24+\ldots & \text { as } \omega \rightarrow 0 \\
(-1)^{x}\left(1-i x \sqrt{4-\omega^{2}}-\left(4-\omega^{2}\right) x^{2} / 2+\ldots\right) & \text { as } \omega \rightarrow \pm 2+i 0
\end{array} \mid x \in \mathbb{N} .\right.
$$

The representations (A.5), (A.7) and (A.9) lead to the following result.

Lemma A.2 (cf [14, Lemma 3.2]) For every $N \in \mathbb{N}$,

$$
e^{i \theta(\omega) x}=1+\sum_{j=1}^{N}\left(\omega^{2}-m^{2}\right)^{j / 2} R^{j}(x)+r_{N}(\omega, x), \quad \omega \rightarrow \pm m+i 0,
$$

where $\left\|r_{N}(\omega, \cdot)\right\|_{\alpha,+}=O\left(\left|\omega^{2}-m^{2}\right|^{(N+1) / 2}\right)$ for $\alpha<-3 / 2-N$. Moreover,

$$
D_{\omega}^{r}\left(e^{i \theta(\omega) x}\right)=\sum_{j=1}^{N} \frac{d^{r}}{d \omega^{r}}\left(\omega^{2}-m^{2}\right)^{j / 2} R^{j}(x)+\tilde{r}_{N}(\omega, x), \quad \omega \rightarrow \pm m+i 0,
$$

where $\left\|\tilde{r}_{N}(\omega, \cdot)\right\|_{\alpha,+}=O\left(\left|\omega^{2}-m^{2}\right|^{(N+1) / 2-r}\right)$ for $\alpha<-3 / 2-N$. The similar representation holds for $\omega \rightarrow \pm \sqrt{m^{2}+4}+i 0$. 
Indeed, the bound (A.10) follows from the following representation for remainder (see formula (A.5)

$$
r_{N}(\omega, x)=\left(\omega^{2}-m^{2}\right)^{(N+1) / 2} \sum_{k=1}^{N+1} b_{k}(\omega, x) x^{k},
$$

where $b_{k}(\omega, x)$ are uniformly bounded for $\omega \rightarrow \pm m+i 0$ and any $x$. In particular,

$$
e^{i \theta(\omega) x}=1+\sqrt{\omega^{2}-m^{2}} R_{0}(\omega, x) \text { as } \omega \rightarrow \pm m+i 0
$$

where $\sup _{|\omega| \leq m+\delta}\left|R_{0}(\omega, x)\right| \leq C|x|$, and $\sup _{|\omega| \leq m+\delta}\left\|R_{0}(\omega, \cdot)\right\|_{\alpha,+} \leq C<\infty$ for $\alpha<-3 / 2$.

\section{A.2 Proof of Theorem 3.3}

To prove (3.6), we use the properties (I)-(III) of $\tilde{K}(x, \omega)$. At first, we rewrite $K(x, t)$ in the form

$$
K(x, t)=\frac{1}{2 \pi} \int_{\Im \omega=\mu>0} e^{-i \omega t} \tilde{K}(x, \omega) d \omega=-\frac{1}{2 \pi} \int_{\Gamma} e^{-i \omega t} \tilde{K}(x, \omega) d \omega, \quad x \in \mathbb{N}, \quad t>0,
$$

where $\Gamma=\left\{|\omega|=R: R>\sqrt{4+m^{2}}\right\}$, and the contour $\Gamma$ is oriented anticlockwise. Since $\tilde{K}(x, \omega)$ is analytic in $\mathbb{C} \backslash \Lambda$, we can vary the integration contour on $\Lambda_{\varepsilon}$, where $\Lambda_{\varepsilon}$ surrounds the segments of $\Lambda$ and belongs to an $\varepsilon$-neighborhood of $\Lambda$ ( $\Lambda_{\varepsilon}$ is oriented anticlockwise). Taking a limit as $\varepsilon \rightarrow 0$, we find

$$
\begin{aligned}
K(x, t) & =\frac{1}{2 \pi} \int_{\Lambda} e^{-i \omega t}(\tilde{K}(x, \omega+i 0)-\tilde{K}(x, \omega-i 0)) d \omega \\
& =\frac{i}{\pi} \int_{\Lambda} e^{-i \omega t} \Im \tilde{K}(x, \omega+i 0) d \omega=\sum_{ \pm} \sum_{j=1}^{2} \frac{i}{\pi} \int_{\Lambda} e^{-i \omega t} P_{j}^{ \pm}(x, \omega) d \omega .
\end{aligned}
$$

Here $P_{j}^{ \pm}(x, \omega):=\zeta_{j}^{ \pm}(\omega) \Im \tilde{K}(x, \omega+i 0), j=1,2$, where $\zeta_{j}^{ \pm}(\omega)$ are smooth functions such that $\sum_{ \pm, j} \zeta_{j}^{ \pm}(\omega)=1, \omega \in \mathbb{R}, \operatorname{supp} \zeta_{1}^{ \pm} \subset \mathcal{O}( \pm m), \operatorname{supp} \zeta_{2}^{ \pm} \subset \mathcal{O}\left( \pm \sqrt{4+m^{2}}\right)(\mathcal{O}(a)$ denotes a neighborhood of the point $\omega=a)$. In the case $m=0$, instead of $\zeta_{1}^{ \pm}\left(P_{1}^{ \pm}\right)$we introduce the function $\zeta_{1}$ (respectively, $\left.P_{1}\right)$ with $\operatorname{supp} \zeta_{1} \subset \mathcal{O}(0)$. By the property (III),

$$
\begin{aligned}
& \left\|P_{1}^{ \pm}(\cdot, \omega)\right\|_{\alpha,+}=O\left(|\omega \mp m|^{1 / 2}\right) \quad \text { if } m \neq 0, \quad\left\|P_{1}(\cdot, \omega)\right\|_{\alpha,+}=O(|\omega|) \quad \text { if } m=0 \\
& \left\|P_{2}^{ \pm}(\cdot, \omega)\right\|_{\alpha,+}=O\left(\left|\omega \mp \sqrt{4+m^{2}}\right|\right)
\end{aligned}
$$

for any $\alpha<-3 / 2$. Therefore, using [13, Lemma 10.2] we obtain

$$
\left\|\int_{\Lambda} e^{-i \omega t} P_{j}^{ \pm}(x, \omega) d \omega\right\|_{\alpha,+}=O\left(|t|^{-3 / 2}\right) \quad \text { as } t \rightarrow \infty, \quad \text { for any } \quad \alpha<-3 / 2 \text {. }
$$

The bound (3.6) follows from (A.11) and (A.12). 


\section{A.3 Existence of solutions}

Lemma A.3 Let $\alpha \in \mathbb{R}, m, \gamma \geq 0$, and let $P$ satisfy the condition (1.5). Then the following assertions hold. (i) For every $Y_{0} \in \mathcal{H}_{\alpha,+}$, the problem (1.1)-(1.3) has a unique solution $Y(t) \in C\left(\mathbb{R}, \mathcal{H}_{\alpha,+}\right)$. Moreover, the operator $U(t): Y_{0} \mapsto Y(t), t \in \mathbb{R}$, is continuous on $\mathcal{H}_{\alpha,+}$, and there exist constants $C, B<\infty$ such that

$$
\|Y(t)\|_{\alpha,+} \leq C e^{B|t|} \quad \text { for } t \in \mathbb{R}
$$

(ii) For $Y_{0} \in \mathcal{H}_{0,+}$, the following identity holds

$$
\mathrm{H}(Y(t))+\gamma \int_{0}^{t}|\dot{u}(0, s)|^{2} d s=\mathrm{H}\left(Y_{0}\right), \quad t \in \mathbb{R}
$$

which implies the estimate $\mathrm{H}(Y(t)) \leq \mathrm{H}\left(Y_{0}\right)$, where $\mathrm{H}(Y(t))$ is the Hamiltonian defined in (1.4). In particular, if $\gamma=0$, the energy $\mathrm{H}(Y(t))$ is conserved and finite.

Proof of Lemma A.3 To prove the existence of $u(x, t)$, it suffices to prove the existence of the solutions $q(t) \equiv q(0, t)$ to the problem (2.6) , (2.8). It follows from the representation (2.1), Lemma 2.4 and formula (3.4). Further, using (3.4), we write (2.6) in the equivalent integral form

$$
q(t)=\int_{0}^{t}\left(\int_{0}^{s} \mathcal{F}(\tau, q(\tau)) d \tau\right) d s+\int_{0}^{t}\left(\int_{0}^{s} z(1, \tau) d \tau-\gamma q(s)\right) d s+C_{0}+C_{1} t, \quad t>0 .
$$

Here $\mathcal{F}(t, q(t)):=-\left(1+m^{2}\right) q(t)+F(q(t))+\int_{0}^{t} K(1, t-s) q(s) d s, C_{0}=q(0) \equiv q_{0}, C_{1}=$ $\dot{q}(0) \equiv p_{0}$. By the bound (3.7), condition (1.5), and the contraction mapping principle, the solution $q(t)$ of (A.15) is unique on a certain interval $t \in[0, \varepsilon)$ with some $\varepsilon>0$ depending on the initial data $\left(q_{0}, p_{0}\right)$. Hence, by (3.4), the solution $q(x, t)$ of the problem (2.5) $-(2.8)$ is unique on a certain interval $t \in[0, \varepsilon)$ with some $\varepsilon>0$ depending on the initial data $Y_{0}$. The existence of $z(x, t)$ is stated in Lemma 2.4. This implies the existence of the local solution $u(x, t)=z(x, t)+q(x, t)$ for sufficiently small $t$. This local solution can be extended to the global solution using the a priori estimate (A.13). Now we verify (A.13). Indeed, by (2.6) and (3.4),

$$
\begin{aligned}
& \frac{1}{2}\left(|\dot{q}(t)|^{2}+\left(m^{2}+1\right)|q(t)|^{2}\right)+P(q(t))+\gamma \int_{0}^{t}|\dot{q}(s)|^{2} d s \\
& =\frac{1}{2}\left(\left|p_{0}\right|^{2}+\left(m^{2}+1\right)\left|q_{0}\right|^{2}\right)+P\left(q_{0}\right)+\int_{0}^{t} \dot{q}(s)\left(z(1, s)-\int_{0}^{s} K(1, s-\tau) q(\tau) d \tau\right) d s .
\end{aligned}
$$

Define $M(t)=\sup _{0 \leq s \leq t}\left(|q(s)|^{2}+|\dot{q}(s)|^{2}\right)$. Then (A.16) and (3.7) yield

$$
M(t) \leq C_{1}+\int_{0}^{t} \sqrt{M(s)}|z(1, s)| d s+C_{2} \int_{0}^{t} M(s) d s, \quad t>0 .
$$

Applying the Gronwall-Bellman integral type inequality (see, for instance, [19]), we find

$$
M(t) \leq e^{C_{2} t}\left(\sqrt{C_{1}}+\frac{1}{2} \int_{0}^{t}|z(1, s)| e^{C_{2} s / 2} d s\right)^{2}, \quad t>0
$$


Since $|z(1, t)| \leq C\langle t\rangle^{\sigma}\left\|Y_{0}\right\|_{\alpha,+}$ (see (C.5) ), we obtain the a priori bound

$$
|q(t)|+|\dot{q}(t)| \leq C e^{B|t|}
$$

with some constants $C, B<\infty$. By (A.2) and (3.4),

$$
\left(\sum_{x \in \mathbb{N}}\langle x\rangle^{2 \alpha}\left(|q(x, t)|^{2}+|\dot{q}(x, t)|^{2}\right)\right)^{1 / 2} \leq C_{1} e^{B|t|}
$$

Thus, the a priori bound (A.13) follows from (2.1), (A.17), (A.18) and (C.5).

\section{Appendix B: Properties of $\tilde{D}(\omega)$ and $\tilde{N}(\omega)$ for $\omega \in \mathbb{C}$}

In this section, we study $\tilde{D}(\omega)$ and $\tilde{N}(\omega)=(\tilde{D}(\omega))^{-1}$. Denote by $\mathbb{C}_{+}\left(\mathbb{C}_{-}\right)$the upper (respectively, lower) half-plane, $\mathbb{C}_{ \pm}=\{\omega \in \mathbb{C}: \pm \Im \omega>0\}$.

Lemma B.4 (i) The function $\tilde{N}(\omega)$ is meromorphic for $\omega \in \mathbb{C} \backslash \Lambda$.

(ii) $|\tilde{N}(\omega)|=O\left(|\omega|^{-2}\right)$ as $|\omega| \rightarrow \infty$.

(iii) $\tilde{D}(\omega) \neq 0$ for all $\omega \in \mathbb{C}_{+}$.

Proof Assertion (i) of the lemma follows from the formula (3.11) and the analyticity of $\tilde{D}(\omega)$ for $\omega \in \mathbb{C} \backslash \Lambda$ (see property (I) of $\tilde{K}(x, \omega)$ in Appendix A). Assertion (ii) follows from (3.11) and (A.1). To prove assertion (iii), we assume contrarily that $\tilde{D}\left(\omega_{0}\right)=0$ for some $\omega_{0} \in \mathbb{C}_{+}$. Hence, the function $u_{*}(x, t)=e^{i \theta\left(\omega_{0}\right) x} e^{-i \omega_{0} t}, x \in \mathbb{Z}_{+}, t \geq 0$, is a solution of the problem (1.1), (1.2) with the initial data $Y_{*}(x)=e^{i \theta\left(\omega_{0}\right) x}\left(1,-i \omega_{0}\right)$. Therefore, the Hamiltonian (1.4) is

$$
\mathrm{H}\left(u_{*}(\cdot, t), \dot{u}_{*}(\cdot, t)\right)=e^{2 t \Im \omega_{0}} \mathrm{H}\left(Y_{*}\right) \quad \text { for all } t>0 \text {, where } \mathrm{H}\left(Y_{*}\right)>0 .
$$

Since $\Im \omega_{0}>0$ and $Y_{*} \in \mathcal{H}_{0,+}$, this exponential growth contradicts the energy estimate (A.14). Hence, $\tilde{D}(\omega) \neq 0$ for any $\omega \in \mathbb{C}_{+}$.

Corollary B.5 If $\gamma=0$, then $\tilde{D}(\omega) \neq 0$ for any $\omega \in \mathbb{C}_{-}$.

Indeed, if $\gamma=0$, then $\bar{D}(\omega)=\tilde{D}(\bar{\omega})$, because $\overline{\theta(\omega)}=-\theta(\bar{\omega})$ for $\omega \in \mathbb{C} \backslash \Lambda$. Therefore, Corollary B.5 follows from item (iii) of Lemma B.4.

Now we study the invertibility of $\tilde{D}(\omega)$ for $\omega \in \mathbb{R}$.

Lemma B.6 Let $\omega \in \mathbb{R}$, and the constants $\gamma, m, \kappa$ satisfy condition $\mathbf{C}$. Then $\tilde{D}(\omega) \neq 0$ for $\omega \in \mathbb{R} \backslash \Lambda$ and $\tilde{D}(\omega \pm i 0) \neq 0$ for $\omega \in \Lambda$.

Proof Step 1: Let $\omega \in \mathbb{R}$ and $|\omega|>\sqrt{4+m^{2}}$. Then $\Re \theta(\omega)= \pm \pi$. Therefore,

$$
\tilde{D}(\omega)=-\omega^{2}+\kappa+1+m^{2}-i \omega \gamma+e^{-\Im \theta(\omega)} \quad \text { with } \Im \theta(\omega)>0 .
$$

Hence, $\Im \tilde{D}(\omega) \neq 0$ iff $\gamma \neq 0$. On the other hand, $\Re \tilde{D}(\omega)=\kappa-2$ for $\omega= \pm \sqrt{4+m^{2}}$ and $\Re \tilde{D}\left(\omega_{1}\right)<\Re \tilde{D}\left(\omega_{2}\right)$ if $\left|\omega_{1}\right|>\left|\omega_{2}\right| \geq \sqrt{4+m^{2}}$. In particular, $\Re \tilde{D}(\omega) \rightarrow-\infty$ as $|\omega| \rightarrow \infty$. Hence, for $|\omega|>\sqrt{4+m^{2}}, \Re \tilde{D}(\omega) \neq 0$ iff $\kappa \leq 2$. Therefore, for such values of $\omega, \tilde{D}(\omega) \neq 0$ iff either $\gamma \neq 0$ or $\gamma=0$ and $\kappa \leq 2$. If $\gamma=0$ and $\kappa>2$, then there exist two points $\pm \omega_{0}$ $\left(\omega_{0}>\sqrt{4+m^{2}}\right)$ such that $\tilde{D}\left( \pm \omega_{0}\right)=0$. 
Step 2: Let $m \neq 0$ and $\omega \in(-m, m)$. Then, $\Re \theta(\omega)=0$ and $e^{i \theta(\omega)} \in\left(e^{i \theta(0)}, e^{i \theta( \pm m)}\right)=$ $\left(4\left(m+\sqrt{4+m^{2}}\right)^{-2}, 1\right)$. Hence,

$$
\Re \tilde{D}(\omega)=-\omega^{2}+\kappa+1+m^{2}-e^{i \theta(\omega)}>\kappa \text { for }|\omega|<m,
$$

and $\Re \tilde{D}( \pm m)=\kappa$. Therefore, $\tilde{D}(\omega) \neq 0$ for any $|\omega|<m$, since $\kappa \geq 0$.

Step 3: Let $\omega \in\left(-\sqrt{4+m^{2}},-m\right) \cup\left(m, \sqrt{4+m^{2}}\right)$. Then $\Re \theta(\omega+i 0) \in(-\pi, 0) \cup(0, \pi)$ and $\Im \theta(\omega+i 0)=0$. Moreover, $\operatorname{sign}(\sin \theta(\omega+i 0))=\operatorname{sign} \omega$. Hence, for $m \neq 0$,

$$
\Im \tilde{D}(\omega+i 0)=-\omega \gamma-\sin \theta(\omega+i 0)=-\operatorname{sign}(\omega)\left(|\omega| \gamma+\frac{1}{2} \sqrt{\omega^{2}-m^{2}} \sqrt{4+m^{2}-\omega^{2}}\right) \neq 0 .
$$

If $m=0$, then for $\omega \in(-2,0) \cup(0,2)$,

$$
\tilde{D}(\omega+i 0)=\kappa-\omega^{2} / 2-i \omega\left(\gamma+\frac{1}{2} \sqrt{4-\omega^{2}}\right) \neq 0 \text { for any } \kappa, \gamma \geq 0 .
$$

Step 4: Since $\tilde{D}(\omega-i 0)=\overline{\tilde{D}(\omega+i 0)}-2 i \omega \gamma$ for $\omega \in \Lambda \backslash \Lambda_{0}$, then

$$
\begin{aligned}
\tilde{D}(\omega-i 0) & =-\omega^{2}+\kappa+1+m^{2}-\cos \theta(\omega+i 0)+i \sin \theta(\omega+i 0)-i \omega \gamma \\
& =\kappa-\left(\omega^{2}-m^{2}\right) / 2+i\left(\operatorname{sign}(\omega) \frac{1}{2} \sqrt{\omega^{2}-m^{2}} \sqrt{4+m^{2}-\omega^{2}}-\omega \gamma\right),
\end{aligned}
$$

for $\omega \in \Lambda \backslash \Lambda_{0}$. Hence, $\tilde{D}(\omega-i 0)=0$ for $\omega \in \Lambda \backslash \Lambda_{0}$ iff

$$
\kappa=\left(\omega^{2}-m^{2}\right) / 2 \text { and } \sqrt{\omega^{2}-m^{2}} \sqrt{4+m^{2}-\omega^{2}}=2|\omega| \gamma, \quad \omega^{2} \in\left(m^{2}, m^{2}+4\right) .
$$

Assume that $\omega$ is a solution of $(\underline{B .1})$. Then, evidently, $\gamma \neq 0$. Put $P:=\omega^{2}-m^{2}$. Hence, $P$ is a solution of the following equation

$$
P^{2}+4 P\left(\gamma^{2}-1\right)+4 m^{2} \gamma^{2}=0 \text { such that } P \in(0,4) .
$$

If $m=0$, then Eqn (B.2) has a unique solution $P=4\left(1-\gamma^{2}\right) \in(0,4)$ iff $\gamma \in(0,1)$. Then, $\kappa=\left(\omega^{2}-m^{2}\right) / 2=P / 2=2\left(1-\gamma^{2}\right)$ by the first equation in (B.1). Thus, if $m=0$, $\kappa=2\left(1-\gamma^{2}\right)$ and $\gamma \in(0,1)$, then there exist two points $\omega= \pm \omega_{*}= \pm 2 \sqrt{1-\gamma^{2}} \in \Lambda \backslash \Lambda_{0}$ such that $\tilde{D}\left(\omega_{*}-i 0\right)=0$.

If $m \neq 0$, then $(\bar{B} .2)$ has a solution iff $\left(\gamma^{2}-1\right)^{2}-m^{2} \gamma^{2} \geq 0$ and $\gamma \in(0,1)$. This is equivalent to the conditions $\gamma^{2}+m \gamma-1 \leq 0$ and $\gamma \in(0,1)$, that coincides with the inequality $\gamma \in\left(0,\left(\sqrt{m^{2}+4}-m\right) / 2\right]$. Therefore, if $m \neq 0$ and $\gamma \in\left(0,\left(\sqrt{m^{2}+4}-m\right) / 2\right]$, then Eqn (B.2) has two solutions

$$
P_{ \pm}=2\left(1-\gamma^{2}\right) \pm 2 \sqrt{\left(1-\gamma^{2}\right)^{2}-m^{2} \gamma^{2}}, \quad P_{ \pm} \in(0,4) .
$$

Hence, $\kappa=\left(\omega^{2}-m^{2}\right) / 2=P_{ \pm} / 2=1-\gamma^{2} \pm \sqrt{\left(1-\gamma^{2}\right)^{2}-m^{2} \gamma^{2}}$.

Thus, there are points $\omega_{*} \in \Lambda \backslash \Lambda_{0}$, in which $\tilde{D}\left(\omega_{*}-i 0\right)=0$, iff $\gamma \neq 0$ and one of the following conditions is fulfilled: (1) $m=0, \kappa=2\left(1-\gamma^{2}\right)$ and $\gamma \in(0,1) ;(2) m \neq 0$, $\kappa=1-\gamma^{2} \pm \sqrt{\left(1-\gamma^{2}\right)^{2}-m^{2} \gamma^{2}}$ and $\gamma \in\left(0,\left(\sqrt{m^{2}+4}-m\right) / 2\right]$. These values of $\kappa, m, \gamma$ are eliminated by the condition $\mathbf{C}$.

Step 5: If $\omega= \pm \sqrt{4+m^{2}}$, then $e^{i \theta(\omega)}=-1$, and $\tilde{D}(\omega)=\kappa-2 \mp i \gamma \sqrt{4+m^{2}}$. Hence,

$$
\tilde{D}\left( \pm \sqrt{4+m^{2}}\right) \neq 0 \text { iff } \gamma \neq 0 \text { or } \gamma=0 \text { and } \kappa \neq 2 \text {. }
$$

If $\omega= \pm m$, then $e^{i \theta(\omega)}=1$, and $\tilde{D}(\omega)=\kappa \mp i \gamma m$. Hence, in the case $m \neq 0, \tilde{D}( \pm m) \neq 0$ iff $\gamma \neq 0$ or $\gamma=0$ and $\kappa \neq 0$. In the case $m=0, \tilde{D}(0)=\kappa \neq 0$ iff $\kappa \neq 0$. Therefore, if Condition $\mathbf{C}$ holds, then $\tilde{D}\left(\omega_{0}\right) \neq 0$ for $\omega_{0} \in \Lambda_{0}$. 
Corollary B.7 If condition $\mathbf{C}$ is not satisfied, then there are points $\omega \in \mathbb{R}$, in which $\tilde{D}(\omega)=$ 0 . For example, $\tilde{D}(0)=0$ in the case $m=\kappa=0$. If $\gamma=\kappa=0$, then $\tilde{D}( \pm m)=0$. If $\gamma=0$ and $\kappa=2$, then $\tilde{D}\left( \pm \sqrt{m^{2}+4}\right)=0$. If $\gamma=0$ and $\kappa>2$, then $\exists \omega_{0}>\sqrt{4+m^{2}}$ such that $\tilde{D}\left( \pm \omega_{0}\right)=0$, and $\tilde{D}^{\prime}\left(\omega_{0}\right)=-2 \omega_{0}(\kappa-1) /\left(2 \kappa+m^{2}-\omega_{0}^{2}\right)<0$.

Now we study the behavior of $\tilde{D}(\omega)$ and $\tilde{N}(\omega)=(\tilde{D}(\omega))^{-1}$ near the points $\omega= \pm m$ and $\omega= \pm \sqrt{4+m^{2}}$ with any $m, \gamma, \kappa \geq 0$. In the neighborhood of the points $\omega= \pm \sqrt{4+m^{2}}$ we use the representation (A.6) and obtain

$$
\begin{aligned}
\tilde{D}(\omega)= & \kappa-2 \mp i \sqrt{4+m^{2}} \gamma-i \sqrt{4+m^{2}-\omega^{2}}+\frac{1}{2}\left(4+m^{2}-\omega^{2}\right)-i\left(\omega \mp \sqrt{4+m^{2}}\right) \gamma \\
& +\frac{i}{8}\left(4+m^{2}-\omega^{2}\right)^{3 / 2}+\ldots, \quad \omega \rightarrow \pm \sqrt{4+m^{2}}, \quad \omega \in \mathbb{C}_{+},
\end{aligned}
$$

where $\operatorname{sgn}\left(\Re \sqrt{m^{2}+4-\omega^{2}}\right)=\operatorname{sgn}(\Re \omega)$. Therefore, if $\gamma \neq 0$ or $\gamma=0$ and $\kappa \neq 2$, then

$$
\tilde{N}(\omega)=(\tilde{D}(\omega))^{-1}=C_{1}+i C_{2} \sqrt{4+m^{2}-\omega^{2}}+\ldots, \quad \omega \rightarrow \pm \sqrt{4+m^{2}}, \quad \omega \in \mathbb{C}_{+},
$$

with $C_{1}=\left(\kappa-2 \mp i \gamma \sqrt{4+m^{2}}\right)^{-1}$ and $C_{2}=C_{1}^{2}$. If $\gamma=0$ and $\kappa=2$, then

$$
\tilde{N}(\omega)=i\left(4+m^{2}-\omega^{2}\right)^{-1 / 2}+\frac{1}{2}-\frac{i}{8}\left(4+m^{2}-\omega^{2}\right)^{1 / 2}+\ldots, \quad \omega \rightarrow \pm \sqrt{4+m^{2}} .
$$

In the neighborhood of the points $\omega= \pm m$ we apply (A.4) (if $m \neq 0)$ and obtain

$$
\tilde{D}(\omega)=\kappa \mp i m \gamma-i \sqrt{\omega^{2}-m^{2}}-i(\omega \mp m) \gamma-\frac{1}{2}\left(\omega^{2}-m^{2}\right)+\frac{i}{8}\left(\omega^{2}-m^{2}\right)^{3 / 2}+\ldots
$$

as $\omega \rightarrow \pm m, \omega \in \mathbb{C}_{+}$, where $\operatorname{sgn}\left(\Re \sqrt{\omega^{2}-m^{2}}\right)=\operatorname{sgn}(\Re \omega)$. In the case $m=0$, (A.8) yields

$$
\tilde{D}(\omega)=\kappa-i \omega(\gamma+1)-\frac{1}{2} \omega^{2}+\frac{i}{8} \omega^{3}+\ldots, \quad \omega \rightarrow 0
$$

Suppose that either $m \gamma \neq 0$ or $\kappa \neq 0$. Then, by (B.6) and (B.7), we obtain

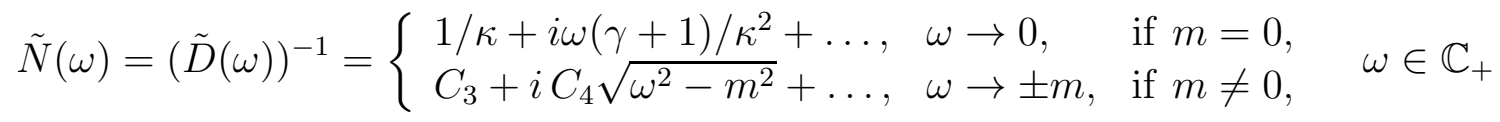

with $C_{3}=(\kappa \mp i m \gamma)^{-1}$ and $C_{4}=C_{3}^{2}$. If $\gamma=\kappa=0$ and $m \neq 0$, then

$$
\tilde{N}(\omega)=i\left(\omega^{2}-m^{2}\right)^{-1 / 2}-\frac{1}{2}-\frac{i}{8}\left(\omega^{2}-m^{2}\right)^{1 / 2} \ldots, \quad \omega \rightarrow \pm m, \quad \omega \in \mathbb{C}_{+} .
$$

If $m=\kappa=0$, then

$$
\tilde{N}(\omega)=\frac{i}{\omega(\gamma+1)}-\frac{1}{2(\gamma+1)^{2}}-\frac{i \omega(\gamma-1)}{8(\gamma+1)^{3}}+\ldots, \quad \omega \rightarrow 0
$$

Since $\tilde{N}(\omega)=(\overline{\tilde{D}(\bar{\omega})}-2 i \omega \gamma)^{-1}$ for $\omega \in \mathbb{C}_{-}$, then the expansion for $\tilde{N}(\omega)$ as $\omega \rightarrow \omega_{0}$ $\left(\omega_{0} \in \Lambda_{0}, \omega \in \mathbb{C}_{-}\right)$can be constructed using (B.3) and (B.6). In particular,

$$
\tilde{N}(\omega+i 0)-\tilde{N}(\omega-i 0)=O\left(\left|\omega^{2}-\omega_{0}^{2}\right|^{1 / 2}\right) \quad \text { as } \omega \rightarrow \omega_{0}, \quad \omega_{0} \in \Lambda_{0} .
$$


Proof of Theorem 3.4 Using Lemma B.4, we vary the integration contour in (3.12):

$$
N(t)=-\frac{1}{2 \pi} \int_{|\omega|=R} e^{-i \omega t} \tilde{N}(\omega) d \omega, \quad t>0,
$$

where $R$ is chosen so large that $\tilde{N}(\omega)$ has no poles in the region $\mathbb{C}_{-} \cap\{|\omega| \geq R\}$. Note that if $\gamma=0$, then $\tilde{N}(\omega)$ has no poles in $\mathbb{C}_{-}$by Corollary B.5. Denote by $\sigma_{j}$ the poles of $\tilde{N}(\omega)$ in $\mathbb{C}_{-}$(if they exist). By Lemmas B.4 and B.6, there exists a $\delta>0$ such that $\tilde{N}(\omega)$ has no poles in the region $\{\omega: \Im \omega \in[-\delta, 0)\}$. Hence, we can rewrite $N(t)$ as

$$
N(t)=-i \sum_{j=1}^{K} \operatorname{Res}_{\omega=\sigma_{j}}\left[e^{-i \omega t} \tilde{N}(\omega)\right]-\frac{1}{2 \pi} \int_{\Lambda_{\varepsilon}} e^{-i \omega t} \tilde{N}(\omega) d \omega, \quad t>0,
$$

where $\varepsilon \in(0, \delta)$, the contour $\Lambda_{\varepsilon}$ surrounds segments of $\Lambda$ and belongs to the $\varepsilon$-neighborhood of $\Lambda$ ( $\Lambda_{\varepsilon}$ is oriented anticlockwise). Passing to a limit as $\varepsilon \rightarrow 0$, we obtain

$$
\begin{aligned}
N(t) & =\frac{1}{2 \pi} \int_{\Lambda} e^{-i \omega t}(\tilde{N}(\omega+i 0)-\tilde{N}(\omega-i 0)) d \omega+o\left(t^{-N}\right) \\
& =\sum_{ \pm} \sum_{j=1}^{2} \frac{1}{2 \pi} \int_{\Lambda} e^{-i \omega t} P_{j}^{ \pm}(\omega) d \omega+o\left(t^{-N}\right), \quad t \rightarrow+\infty, \quad \text { with any } N>0 .
\end{aligned}
$$

Here $P_{j}^{ \pm}(\omega):=\zeta_{j}^{ \pm}(\omega)(\tilde{N}(\omega+i 0)-\tilde{N}(\omega-i 0))$, where $\zeta_{j}^{ \pm}(\omega)$ are as in (A.11). Then (B.11) implies the bound (3.13) with $k=0$. Here we use the following estimate

$$
\left|\int_{\mathbb{R}} \zeta(\omega) e^{-i \omega t}\left(a^{2}-\omega^{2}\right)^{1 / 2} d \omega\right| \leq C(1+t)^{-3 / 2} \text { as } t \rightarrow+\infty,
$$

where $\zeta(\omega)$ is a smooth function, and $\zeta(\omega)=1$ for $|\omega-a| \leq \delta$ with some $\delta>0$ (see, for example, [21, Lemma 2]). The bound (3.13) with $k=1,2$ can be proved in a similar way.

Remark B.8 Now we study the asymptotics of $N(t)$ as $\rightarrow+\infty$ in the case when condition $\mathbf{C}$ is not fulfilled. Assume first that $\gamma=\kappa=0$ and $m \neq 0$. Then $\tilde{N}(\omega+i 0)-\tilde{N}(\omega-i 0)=$ $2 i \Im \tilde{N}(\omega+i 0)$, and $\tilde{N}(\omega)$ has no poles in $\mathbb{C}_{-}$. Introduce the circles $c_{ \pm}, c_{ \pm}=\{|\omega \mp m|=\varepsilon\}$, with some $\varepsilon \in(0, m)$. We change the integration contour in (B.12) on $\Gamma_{\varepsilon}:=\cup_{ \pm} c_{ \pm} \cup_{j} \gamma_{j}$, where $\gamma_{j}, j=1,2,3$, stand for the segments of the real axis connecting the circles $c_{ \pm}$and passing in two directions, $\gamma_{1}=\left[-\sqrt{m^{2}+4},-m-\varepsilon\right], \gamma_{2}=[-m+\varepsilon, m-\varepsilon], \gamma_{3}=\left[m+\varepsilon, \sqrt{m^{2}+4}\right]$. Using the Cauchy theorem and Lemma B.4, we find

$$
N(t)=-\frac{1}{2 \pi} \int_{c_{-} \cup c_{+}} e^{-i \omega t} \tilde{N}(\omega) d \omega+\sum_{j=1}^{3} \frac{i}{\pi} \int_{\gamma_{j}} e^{-i \omega t} \Im \tilde{N}(\omega+i 0) d \omega .
$$

Applying representations (B.4) and (B.9) and the well-known estimate (see, for example, [21])

$$
-\frac{1}{2 \pi} \int_{|\omega|=m+1} e^{-i \omega t}\left(\omega^{2}-m^{2}\right)^{-1 / 2} d \omega=i \sqrt{\frac{2}{\pi m t}} \cos (m t-\pi / 4)+O\left(t^{-3 / 2}\right), \quad t \rightarrow \infty,
$$

we obtain that $N(t)=-(2 /(\pi m))^{1 / 2} t^{-1 / 2} \cos (m t-\pi / 4)+O\left(t^{-3 / 2}\right)$ as $t \rightarrow \infty$. 
Similarly, if $\gamma=0$ and $\kappa=2$, then ( $(\overline{\mathrm{B} .5})$ and $(\underline{\mathrm{B} .8})$ give the bound $|N(t)| \leq C\langle t\rangle^{-1 / 2}$.

In the case $\gamma=0$ and $\kappa>2, \tilde{N}(\omega)$ has two simple poles in the points $\pm \omega_{0}$ with $\omega_{0}>\sqrt{4+m^{2}}$. Then, calculating the rescue of the function $e^{-i \omega t} \tilde{N}(\omega)$ in these points, we have $N(t) \sim C_{1} \sin \omega_{0} t+O\left(t^{-3 / 2}\right)$ as $t \rightarrow+\infty$ with some constant $C_{1} \neq 0$.

If $m=\kappa=0$, then we use formulas $(\underline{\mathrm{B} .10})$ and $(\underline{\mathrm{B} .4})$, calculate the rescue of $\tilde{N}(\omega)$ in the point $\omega=0$ and obtain $N(t)=(\gamma+1)^{-1}+O\left(t^{-3 / 2}\right)$ as $t \rightarrow+\infty$.

Finally, let one of the following two conditions hold (see condition $\mathbf{C}$ ):

(1) $m=0, \kappa=2\left(1-\gamma^{2}\right)$ and $\gamma \in(0,1)$

(2) $m \neq 0, \kappa=1-\gamma^{2} \pm \sqrt{\left(1-\gamma^{2}\right)^{2}-m^{2} \gamma^{2}}$ and $\gamma \in\left(0,\left(\sqrt{m^{2}+4}-m\right) / 2\right]$

Then there exist points $\omega_{*} \in \Lambda \backslash \Lambda_{0}$ such that $\tilde{D}\left(\omega_{*}-i 0\right)=0$ (see Step 4 in the proof of Lemma B.6). Hence, $N(t)=C \sin \left(\omega_{*} t\right)+O\left(t^{-3 / 2}\right)$ as $t \rightarrow \infty$ with some constant $C \neq 0$.

\section{Appendix C: Zero boundary condition}

Consider the following initial-boundary value problem on the half-line:

$$
\left\{\begin{array}{l}
\ddot{u}(x, t)=\left(\Delta_{L}-m^{2}\right) u(x, t), \quad x \in \mathbb{N}, \quad t \in \mathbb{R}, \\
u(0, t)=0, \\
u(x, 0)=u_{0}(x), \quad \dot{u}(x, 0)=v_{0}(x), \quad x \in \mathbb{N} .
\end{array}\right.
$$

In [5], we have proved the convergence to equilibrium for the harmonic crystals on the halfspace in any dimension with zero boundary condition. However, the one-dimensional case with $m=0$ was not considered. Therefore, we outline the strategy of the proof of Theorem 2.8 .

At first, we rewrite the problem (C.1) in the more general form:

$$
\left\{\begin{array}{l}
\ddot{u}(x, t)=-\sum_{x^{\prime} \geq 0}\left(V\left(x-x^{\prime}\right)-V\left(x+x^{\prime}\right)\right) u\left(x^{\prime}, t\right), \quad x \in \mathbb{N}, \quad t \in \mathbb{R}, \\
u(0, t)=0, \\
u(x, 0)=u_{0}(x), \quad \dot{u}(x, 0)=v_{0}(x), \quad x \in \mathbb{N} .
\end{array}\right.
$$

We assume that $u_{0}(0)=v_{0}(0)=0$. We impose the following conditions $\mathbf{V} \mathbf{1}-\mathbf{V} 5$ on the interaction function $V$.

V1 There exist positive constants $C$ and $\beta$ such that $|V(x)| \leq C e^{-\beta|x|}$ for $x \in \mathbb{Z}$.

V2 $V(x)$ is real and even, i.e., $V(-x)=V(x) \in \mathbb{R}, x \in \mathbb{Z}$.

Conditions $\mathbf{V} 1$ and $\mathbf{V} 2$ imply that $\hat{V}(\theta)$ is a real-analytic function of $\theta \in \mathbb{T}$.

V3 $\hat{V}(\theta) \geq 0$ for every $\theta \in \mathbb{T}$.

Let us define the real-valued nonnegative function, $\phi(\theta)=(\hat{V}(\theta))^{1 / 2} \geq 0$. $\phi(\theta)$ can be chosen as the real-analytic function in $\mathbb{T} \backslash \mathcal{C}_{*}$, where the Lebesgue measure of a set $\mathcal{C}_{*}$ is zero (see Lemma 2.2 in [2]).

V4 $\phi^{\prime \prime}(\theta)$ does not vanish identically on $\theta \in \mathbb{T} \backslash \mathcal{C}_{*}$.

V5 $\hat{V}(\theta) \neq 0$ for all $\theta \neq 0$, and $\hat{V}(\theta) \geq C \theta^{2}$ as $\theta \rightarrow 0$ with some positive constant $C$. 
Conditions V1-V5 are fulfilled, for example, in the case when $V(x)$ has a form

$$
V( \pm 1)=-1, \quad V(x)=0 \text { for }|x| \geq 2, \quad \text { and } \quad V(0)=2+m^{2} \text { with } m \geq 0 .
$$

Then $\phi(\theta)=\sqrt{2-2 \cos \theta+m^{2}}$. If $m \neq 0$, then $\mathcal{C}_{*}=\emptyset$. If $m=0$, then $\phi(\theta)=2|\sin (\theta / 2)|$ and $\mathcal{C}_{*}=\{0\}$. Furthermore, in the case when $V(x)$ is of the form (C.3), the problem (C.2) becomes (C.1).

Write $Z(x, t)=\left(Z^{0}(x, t), Z^{1}(x, t)\right) \equiv(u(x, t), \dot{u}(x, t)), Z_{0}(x)=\left(u_{0}(x), v_{0}(x)\right)$. The existence of dynamics is stated by the following lemma which is proved as in [5].

Lemma C.1 Let $\alpha \in \mathbb{R}$ and conditions $\mathbf{V} 1$ and $\mathbf{V} 2$ hold. For any $Z_{0} \in \mathcal{H}_{\alpha,+}$, there exists a unique solution $Z(\cdot, t) \in \mathcal{H}_{\alpha,+}$ to problem (C.2). Moreover, the operator $U_{0}(t): Z_{0} \rightarrow Z(\cdot, t)$ is continuous in $\mathcal{H}_{\alpha,+}$.

Indeed, by condition V2, the solution of problem ([C.2) can be represented as the restriction of the solution to the Cauchy problem with odd initial data on the half-line,

$$
Z^{i}(x, t)=\sum_{y \in \mathbb{Z}} \mathcal{G}_{t}^{i j}(x-y) Z_{\text {odd }}^{j}(y), \quad x \in \mathbb{Z}_{+}, \quad i=0,1 .
$$

Here $\mathcal{G}_{t}(x)=F_{\theta \rightarrow x}^{-1}\left[e^{\hat{\mathcal{A}}(\theta) t}\right]$ with $\hat{\mathcal{A}}(\theta)=\left(\begin{array}{cc}0 & 1 \\ \hat{V}(\theta) & 0\end{array}\right)$ (see also formulas (2.11), (2.12) with $\left.\phi(\theta)=(\hat{V}(\theta))^{1 / 2}\right)$, and by definition, $Z_{\text {odd }}(x)=Z_{0}(x)$ for $x>0, Z_{\text {odd }}(0)=0$, and $Z_{\text {odd }}(x)=$ $-Z_{0}(-x)$ for $x<0$. Then the solution $Z(x, t)$ of problem (C.2) is of a form

$$
Z^{i}(x, t)=\sum_{y \in \mathbb{N}}\left(\mathcal{G}_{t}^{i j}(x-y)-\mathcal{G}_{t}^{i j}(x+y)\right) Z_{0}^{j}(y), \quad x \in \mathbb{Z}_{+}, \quad i=0,1 .
$$

Furthermore, the following bound holds,

$$
\left\|U_{0}(t) Z_{0}\right\|_{\alpha,+} \leq C\langle t\rangle^{\sigma}\left\|Z_{0}\right\|_{\alpha,+},
$$

with some constants $C=C(\alpha), \sigma=\sigma(\alpha)<\infty$.

Denote by $\nu_{0}$ a Borel probability measure on $\mathcal{H}_{\alpha,+}$ giving the distribution of $Z_{0}$. We impose conditions S1-S4 on $\nu_{0}$. Let $\nu_{t}, t \in \mathbb{R}$, denote the distribution of the solution $Z(x, t)=U_{0}(t) Z_{0}$ (see Definition 2.5). Then the following theorem holds (cf Theorem 2.8).

Theorem C.2 Let conditions S1-S4 and V1-V5 hold, and let $\alpha<-1 / 2$ if $\hat{V}(0) \neq 0$, and $\alpha<-1$ if $\hat{V}(0)=0$. Then the following assertions are true.

(i) The measures $\nu_{t}$ weakly converge on the space $\mathcal{H}_{\alpha,+}$ as $t \rightarrow \infty$. Moreover, the limit measure $\nu_{\infty}$ is Gaussian on $\mathcal{H}_{\alpha,+}$ with the correlation matrix of the form (2.14)-(2.15) with $\phi(\theta)=(\hat{V}(\theta))^{1 / 2}$.

(ii) The correlation functions of $\nu_{t}$ converge to a limit as $t \rightarrow \infty$, i.e., (2.13) holds.

The derivation of this theorem is based on the proof of the compactness of the measures family $\left\{\nu_{t}, t \in \mathbb{R}\right\}$ and the convergence of the characteristic functionals. Below we prove only the compactness. The convergence of the characteristic functionals and correlation functions can be proved using the technique from [5]. The compactness follows from the bound (C.6) below by the Prokhorov compactness theorem [22, Lemma II.3.1] and by a method used in [22, Theorem XII.5.2]. 
Lemma C.3 Let all assumptions of Theorem C.2 be fulfilled. Then

$$
\sup _{t \geq 0} \mathbb{E}_{0}\left(\left\|U_{0}(t) Z_{0}\right\|_{\alpha,+}^{2}\right)<\infty .
$$

Proof. By Definition 2.1,

$$
\mathbb{E}_{0}\left(\|Z(\cdot, t)\|_{\alpha,+}^{2}\right)=\sum_{z \in \mathbb{Z}_{+}}\left(1+|z|^{2}\right)^{\alpha}\left(Q_{t}^{00}(z, z)+Q_{t}^{11}(z, z)\right) .
$$

The representation (C.4) gives

$$
Q_{t}^{i j}\left(z, z^{\prime}\right)=\mathbb{E}_{0}\left(Z^{i}(z, t) \otimes Z^{j}\left(z^{\prime}, t\right)\right)=\left\langle Q_{0}\left(y, y^{\prime}\right), \mathbf{G}_{z}^{i}(y, t) \otimes \mathbf{G}_{z^{\prime}}^{j}\left(y^{\prime}, t\right)\right\rangle_{+},
$$

where $\mathbf{G}_{z}^{i}(y, t)$ is defined in (4.1). If $\hat{V}(0) \neq 0$, then the Parseval identity and formula (2.12) with $\phi(\theta)=(\hat{V}(\theta))^{1 / 2}$ yield

$$
\left\|\mathbf{G}_{z}^{i}(\cdot, t)\right\|_{l^{2}}^{2} \leq C \int_{\mathbb{T}}\left(\left|\hat{\mathcal{G}}_{t}^{i 0}(\theta)\right|^{2}+\left|\hat{\mathcal{G}}_{t}^{i 1}(\theta)\right|^{2}\right) \sin ^{2}(z \theta) d \theta \leq C_{0}<\infty .
$$

By conditions S1, S2 and $\mathbf{S 4}$, the bound (2.9) holds. Therefore,

$$
\begin{aligned}
\left|Q_{t}^{i j}\left(z, z^{\prime}\right)\right| & =\left|\left\langle Q_{0}\left(y, y^{\prime}\right), \mathbf{G}_{z}^{i}(y, t) \otimes \mathbf{G}_{z^{\prime}}^{j}\left(y^{\prime}, t\right)\right\rangle_{+}\right| \\
& \leq C\left\|\mathbf{G}_{z}^{i}(\cdot, t)\right\|_{0,+}\left\|\mathbf{G}_{z^{\prime}}^{j}(\cdot, t)\right\|_{0,+} \leq C_{1}<\infty,
\end{aligned}
$$

where the constant $C_{1}$ does not depend on $z, z^{\prime} \in \mathbb{Z}_{+}$and $t \in \mathbb{R}$. Therefore, (C.6) follows from (C.7) and (C.9), since $\alpha<-1 / 2$.

If $\hat{V}(0)=0$, then $\hat{V}(\theta) \geq C \theta^{2}$ as $\theta \rightarrow 0$. The estimate (C.8) with $i=1$ remains true. Then $\left|Q_{t}^{11}\left(z, z^{\prime}\right)\right| \leq C<\infty$, by (C.9). However, since $\hat{\mathcal{G}}_{t}^{01}(\theta)=\sin (\phi(\theta) t) / \phi(\theta)$, we have

$$
\left\|\mathbf{G}_{z}^{0}(\cdot, t)\right\|_{l^{2}}^{2} \leq C+C_{1} \int_{\mathbb{T}} \frac{\sin ^{2}(\phi(\theta) t)}{\hat{V}(\theta)} \sin ^{2}(z \theta) d \theta \leq C+C_{2} \int_{\mathcal{O}(0)} \frac{\sin ^{2}(z \theta)}{\sin ^{2}(\theta)} d \theta \leq C+C_{3}|z|,
$$

uniformly on $t \in \mathbb{R}$. Hence, $\left|Q_{t}^{00}(z, z)\right| \leq C\left\|\mathbf{G}_{z}^{0}(\cdot, t)\right\|_{0,+}^{2} \leq C_{1}+C_{2}|z|$. Therefore, for any $\alpha<-1$, we have $\mathbb{E}_{0}\left(\|Z(\cdot, t)\|_{\alpha,+}^{2}\right) \leq \sum_{z \in \mathbb{Z}_{+}}\langle z\rangle^{2 \alpha}\left(C_{1}+C_{2}|z|\right)<\infty$.

\section{References}

[1] Boldrighini, C., Pellegrinotti, A., and Triolo, L., "Convergence to stationary states for infinite harmonic systems," J. Stat. Phys. 30, 123-155 (1983).

[2] Dudnikova, T.V., Komech, A.I., and Spohn, H., "On the convergence to statistical equilibrium for harmonic crystals," J. Math. Phys. 44, 2596-2620 (2003). e-print arXiv:math-ph/0210039.

[3] Dudnikova, T.V., Komech, A.I., and Mauser, N.J., "On two-temperature problem for harmonic crystals," J. Stat. Phys. 114, no.3/4, 1035-1083 (2004). e-print arXiv:math-ph/0508048 
[4] Dudnikova, T.V. and Komech, A.I., "On the convergence to a statistical equilibrium in the crystal coupled to a scalar field," Russian J. Math. Phys. 12 (3), 301-325 (2005). e-print ArXiv:math-ph/0508053.

[5] Dudnikova, T.V., "On the asymptotical normality of statistical solutions for harmonic crystals in half-space," Russian J. Math. Phys. 15, no.4, 460-472 (2008). e-print arXiv:0905.3472.

[6] Dudnikova, T.V., "Long-time asymptotics of solutions to a Hamiltonian system on a lattice," Journal of Mathematical Sciences 219 (1), 69-86 (2016). [English transl.: Problems in Mathematical Analysis 85, 69-82 (2016)].

[7] Eckmann, J.-P., Pillet C.-A., and Rey-Bellet, L., "Non-equilibrium statistical mechanics of anharmonic chains coupled to two heat baths at different temperatures," Commun. Math. Phys. 201, 657-697 (1999).

[8] G. Gripenberg, S.-O. Londen, O. Staffans, Volterra Integral and Functional Equations, vol. 34, in: Encyclopedia of Mathematics and its Applications, Cambridge University Press, Cambridge (1990).

[9] Ibragimov, I.A. and Linnik, Yu.V., Independent and Stationary Sequences of Random Variables, edited by J. F. C. Kingman (Wolters-Noordhoff, Groningen, 1971).

[10] Islami, H. and Vainberg, B., "Large time behavoir of the solutions to difference wave operators," Commun. in Partial Dif. Eq. 31 (3), 397-416 (2006).

[11] Jakšić, V. and Pillet, C.-A., "Ergodic properties of the non-Markovian Langevin equation," Lett. Math. Phys. 41 (1), 49-57 (1997).

[12] Jakšić, V. and Pillet, C.-A., "Ergodic properties of classical dissipative systems. I," Acta Math. 181 (2), 245-282 (1998).

[13] Jensen, A. and Kato, T., "Spectral properties of Schrodinger operators and time-decay of the wave functions," Duke Math. J. 46, 583-611 (1979).

[14] Komech, A.I., Kopylova, E.A., and Kunze, M., "Dispersive estimates for 1D discrete Schrodinger and Klein-Gordon equations," Applicable Anal. 85 (12), 1487-1508 (2006).

[15] I.-G. E. Kordonis, Ch. G. Philos, The behavior of solutions of linear integro-differential equations with unbounded delay, Computers and Mathematics with Appl. 38, 45-50 (1999).

[16] S. Murakami, Exponential asymptotic stability for scalar linear Volterra equations, Differential and Integral Equations 4, 519-525 (1991).

[17] Y. Nino, S. Murakami, Stability properties of linear Volterra equations, J. Differential Equations 89, 121-137 (1991).

[18] M. Ottobre and G. A. Pavliotis, Asymptotic analysis for the generalized Langevin equation, Nonlineartity 24 (5), 1629-1653 (2011). 
[19] Pachpatte, B.G., "On some generalizations of Bellman's lemma," J. Math. Anal. Appl. 51, 141-150 (1975).

[20] Spohn, H. and Lebowitz, J.L., "Stationary non equilibrium states of infinite harmonic systems," Comm. Math. Phys. 54, 97-120 (1977).

[21] Vainberg, B., "Behavior for large time of solutions of the Klein-Gordon equation," Trans. Moscow Math. Soc. 30, 139-158 (1974).

[22] Vishik, M.I. and Fursikov, A.V., Mathematical Problems of Statistical Hydromechanics (Kluwer Academic, New York, 1988). 